\title{
NGC 3105: a young open cluster with low metallicity ${ }^{\star \star \star}$
}

\author{
J. Alonso-Santiago ${ }^{1}$, A. Marco ${ }^{1}$, I. Negueruela ${ }^{1}$, H. M. Tabernero ${ }^{1}$, N. Castro ${ }^{2}$, \\ V. A. McBride ${ }^{3,4,5}$, and A. F. Rajoelimanana ${ }^{4,5,6}$
}

\author{
1 Departamento de Física, Ingeniería de Sistemas y Teoría de la Señal, Escuela Politécnica Superior, Universidad de Alicante, \\ Carretera de San Vicente del Raspeig s/n, 03690 Alicante, Spain \\ e-mail: javier.alonso@ua.es \\ 2 Department of Astronomy, University of Michigan, 1085 S. University Avenue, Ann Arbor, MI 48109-1107, USA \\ 3 Office of Astronomy for Development, International Astronomical Union, Cape Town, South Africa \\ 4 South African Astronomical Observatory, PO Box 9, Observatory 7935, South Africa \\ 5 Department of Astronomy, University of Cape Town, Private Bag X3, Rondebosch 7701, South Africa \\ ${ }^{6}$ Department of Physics, University of the Free State, PO Box 339, Bloemfontein 9300, South Africa
}

Received 22 March 2018 / Accepted 30 April 2018

\begin{abstract}
Context. NGC 3105 is a young open cluster hosting blue, yellow, and red supergiants. This rare combination makes it an excellent laboratory for constraining evolutionary models of high-mass stars. It has been poorly studied, and the fundamental parameters such as its age or distance are not well defined.

Aims. We intend to characterise in an accurate way the cluster and its evolved stars, for which we derive for the first time atmospheric parameters and chemical abundances.

Methods. We performed a complete analysis combining $U B V R$ photometry with spectroscopy. We obtained spectra with classification purposes for 14 blue stars and high-resolution spectroscopy for an in-depth analysis of the six other evolved stars.

Results. We identify $126 \mathrm{~B}$-type likely members within a radius of $2.7 \pm 0.6$ arcmin, which implies an initial mass, $M_{\mathrm{cl}} \approx 4100 M_{\odot}$. We find a distance of $7.2 \pm 0.7 \mathrm{kpc}$ for NGC 3105 , placing it at $R_{\mathrm{GC}}=10.0 \pm 1.2 \mathrm{kpc}$. Isochrone fitting supports an age of $28 \pm 6 \mathrm{Ma}$, implying masses around $9.5 M_{\odot}$ for the supergiants. A high fraction of Be stars $(\approx 25 \%)$ is found at the top of the main sequence down to spectral type b3. From the spectral analysis we estimate for the cluster an average $v_{\text {rad }}=+46.9 \pm 0.9 \mathrm{~km} \mathrm{~s}^{-1}$ and a low metallicity, $[\mathrm{Fe} / \mathrm{H}]=-0.29 \pm 0.22$. We also have determined, for the first time, chemical abundances for $\mathrm{Li}, \mathrm{O}, \mathrm{Na}, \mathrm{Mg}, \mathrm{Si}, \mathrm{Ca}, \mathrm{Ti}, \mathrm{Ni}, \mathrm{Rb}, \mathrm{Y}$, and $\mathrm{Ba}$ for the evolved stars. The chemical composition of the cluster is consistent with that of the Galactic thin disc. An overabundance of $\mathrm{Ba}$ is found, supporting the enhanced $s$-process.

Conclusions. NGC 3105 has a low metallicity for its Galactocentric distance, comparable to typical LMC stars. It is a valuable spiral tracer in a very distant region of the Carina-Sagittarius spiral arm, a poorly known part of the Galaxy. As one of the few Galactic clusters containing blue, yellow, and red supergiants, it is massive enough to serve as a test bed for theoretical evolutionary models close to the boundary between intermediate- and high-mass stars.
\end{abstract}

Key words. open clusters and associations: individual: NGC 3105 - Hertzsprung-Russell and C-M diagrams - stars: fundamental parameters - stars: abundances - stars: late-type - stars: emission-line, Be

\section{Introduction}

Red supergiants (RSGs) are believed to correspond to the Hecore burning phase of stars with masses between $\approx 8$ and $25 M_{\odot}$ (Levesque et al. 2005). They represent a very important population, because they are easy to identify and, being extremely bright in the infrared $\left(M_{K}\right.$ up to $\left.\sim-11\right)$, can be seen through very heavy obscuration. Their numbers and properties are a fundamental test for models of high-mass star evolution (Levesque et al. 2005; Meynet et al. 2011). Over the last years, several clusters of RSGs have been found in the inner Galaxy

^ This research is partially based on observations made with the New Technology Telescope under programme 090.D-0302(A) and the MPG/ESO $2.2 \mathrm{~m}$ Telescope under programme 095.A-9020(A). Both telescopes are operated at La Silla Observatory (Chile) by the European Southern Observatory.

$\star \star$ Full Tables A.1 and A.2 are only available at the CDS via anonymous ftp to cdsarc.u-strasbg.fr $(130.79 .128 .5)$ or via http://cdsarc.u-strasbg.fr/viz-bin/qcat?]/A+A/616/A124
(Davies et al. 2007; Clark et al. 2009b; Negueruela et al. 2011; González-Fernández \& Negueruela 2012). These red supergiant clusters (RSGCs) are concentrations of RSGs (between 8 and 26 such objects) in regions of very high obscuration. The high extinction has so far prevented the study of cluster members other than the RSGs, rendering the determination of cluster parameters very insecure (Davies et al. 2007). Clusters with similar RSG populations and much lower obscuration are very rare, with VdBH 222 (Marco et al. 2014) the only example known until now. The lower obscuration allows the detection of the intrinsically blue population and thus much stronger constraints on the cluster mass.

The RSG phase is very short compared to the lifetime of the stars; RSGs are thus scarce. Most well-studied young open clusters in the 10-25 Ma age range have at most two RSGs (and in many cases none; Eggenberger et al. 2002). However, RSGCs contain so many because they are more massive than typical open clusters in the solar neighbourhood. Population synthesis simulations using a Kroupa IMF and sophisticated modelling predict that we will find on average $5 \pm 2$ RSGs in a $10000 M_{\odot}$ cluster (Clark et al. 2009a). Based on this, Stephenson 2, with 
26 RSGs, would have $M_{\mathrm{cl}} \approx 50000 M_{\odot}$; however, these values are highly tentative because the distances to the RSGCs have been calculated via indirect methods such as radial velocities (Davies et al. 2007) or average extinction (Negueruela et al. 2011). Such methods imply ages around $20 \mathrm{Ma}$ for most of the clusters. Observations reveal much random variation in the number of RSGs. A cluster as massive as $\mathrm{h} \operatorname{Per}\left(M_{\mathrm{cl}} \approx 7000 M_{\odot}\right)$ contains no RSGs, even though there are 5 RSGs within the halo surrounding it and its twin $\chi$ Per, an area that may perhaps contain $20000 M_{\odot}$ (Slesnick et al. 2002). In contrast, the only moderate-extinction cluster known to contain 5 RSGs, NGC 7419, has a similar mass $\approx 7000 M_{\odot}$ (Marco \& Negueruela 2013). This cluster presents some peculiarities that could also be due to statistical fluctuations: it lacks blue supergiants (BSGs), while the fraction of BSGs to RGSs is $>1$ in the overall Milky Way cluster population (Eggenberger et al. 2002); it also presents a very high fraction (the highest known) of Be stars (stars with emission lines). On the other hand, these characteristics, as well as the ratio of RSGs to total mass, might vary from cluster to cluster according to unknown physical properties (binarity, average rotational velocity, etc.).

NGC 3105 is a poorly studied, faint and compact open cluster located in the constellation of Vela $[\alpha(2000)=$ $\left.10^{\mathrm{h}} 00^{\mathrm{m}} 39^{\mathrm{s}}, \delta(2000)=-54^{\circ} 47^{\prime} 18^{\prime \prime} ; \ell=279.92, b=0.26\right]$. Moffat \& Fitzgerald (1974) performed the first study of NGC 3105 employing photographic and photoelectric $U B V$ photometry. Later, Fitzgerald et al. (1977) upgraded this study providing more photoelectric $U B V$ observations and slit spectroscopy for a few stars. Both works agree on the colour excess, $E(B-V) \approx 1.1$, but differ significantly when estimating the distance $(8.0 \pm 1.5 \mathrm{kpc}$ and $5.5 \pm 0.8 \mathrm{kpc}$, respectively). In any case, the reddening to NGC 3105 is quite low for such high distances. Between the two studies, 34 stars were measured photoelectrically. Fitzgerald et al. (1977) identified 15 likely blue members inside a cluster radius of 0'.9 and 7 other likely members at higher distances from the centre. They also identified five candidate yellow and red supergiants and some emission-line star candidates. They give a spectral type at turn-off of B2, slightly later than their previous estimate (Moffat \& Fitzgerald 1974) of b1 ${ }^{1}$, which explains the shorter distance.

Further work on NGC 3105 is scarce. There are only two papers reporting modern CCD observations. Sagar et al. (2001) took $B V R I \mathrm{CCD}$ photometry down to $V \approx 18 \mathrm{mag}$, finding 37 likely members. In combination with the $U$ values from Fitzgerald et al. (1977), and obtaining a similar reddening, they estimated an even greater distance of $9.5 \pm 1.5 \mathrm{kpc}$. More recently, Paunzen et al. (2005) used CCD photometry in the $\Delta a$ photometric system to look for peculiar stars. They placed the cluster at $8.53 \pm 1.03 \mathrm{kpc}$, estimating a slightly lower reddening of $E(B-V)=0.95 \pm 0.02$ from the 48 cluster members selected.

The two CCD studies agree on an age of around 20-25 Ma, which, despite the discrepancy in the distance determination, is compatible with the earliest spectral type on the main sequence (MS) around B2 found by Fitzgerald et al. (1977). At this age, the evolved stars should have masses around $12 M_{\odot}$, comparable to the $\approx 12-16 M_{\odot}$ for the RSGs found in the RSGCs.

Recently, Marco et al. (2013) carried out spectroscopic observations of the brightest stars of the cluster, and found two blue supergiants. With this find, NGC 3105 acquires a very high

\footnotetext{
1 We follow the classical notation which distinguishes spectral types derived via spectroscopy (capital letters) from those derived via photometry (lowercase letters).
}

Table 1. Log of the photometric observations for NGC 3105 taken at the NTT.

\begin{tabular}{lccc}
\hline \hline NGC 3105 & \multicolumn{2}{c}{$\begin{array}{c}\text { RA }=10^{\mathrm{h}} 00^{\mathrm{m}} 39^{\mathrm{s}} \text { Dec }=-54^{\circ} 47^{\prime} 18^{\prime \prime} \\
(\mathrm{J} 2000)\end{array}$} & $\begin{array}{c}(\mathrm{J} 2000) \\
\text { Filter }\end{array}$ \\
\hline \multicolumn{3}{c}{ Exposure times $(\mathrm{s})$} \\
\hline$U$ & Short & Intermediate & Long \\
$B$ & 60 & 500 & 1800,2400 \\
$V$ & 12,24 & 100 & $400,600,800$ \\
$R$ & $3,4,6$ & 18 & 90,100 \\
& 1,3 & 9 & 12,15 \\
\hline
\end{tabular}

intrinsic astrophysical interest. On the one hand, the co-existence of evolved high-mass stars in the blue, yellow, and red supergiant phase is a very rare occurrence because of the short duration of these phases. Only the starburst cluster Westerlund 1 has a full set of evolved high-mass stars (Clark et al. 2005). However, at an age of around $5 \mathrm{Ma}$, Westerlund 1 probes the evolution of stars with $M_{*} \approx 35-40 M_{\odot}$, while NGC 3105 would sample much lower masses. On the other hand, NGC 3105 represents a good template for RSGCs. It is rather less reddened than NGC 7419 and hosts BSGs, which NGC 7419 does not. In addition, it is a valuable spiral tracer in a distant and poorly known part of the Galaxy.

In this work we want to improve the characterisation of the cluster by accurately determining the distance and the other parameters such as age, size, or mass. Morever, our main goal is to obtain the stellar properties of the cool supergiants, atmospheric parameters and chemical abundances, in order to perform the most complete study of the cluster to date. To carry out these tasks, we have the deepest $U B V R$ photometry currently available and spectroscopy for all the bright stars in the field, including high-resolution spectra of the cool supergiants.

\section{Observations and data}

\subsection{Optical photometry}

We obtained $U B V R$ photometry of NGC 3105 in visitor mode using the ESO Faint Object Spectrograph and Camera (EFOSC2) on the NTT $3.6 \mathrm{~m}$ telescope at the La Silla Observatory (Chile) during three consecutive nights in 2013, from January 31 to February 02. EFOSC2 (Buzzoni et al. 1984) covers a field of view of $4 !^{\prime} 1 \times 4$.'1 with a pixel scale of $00^{\prime} .24$ pixel $^{-1}$. Images of NGC 3105 were taken in several series of different exposure times in each filter (see Table 1) to achieve accurate photometry for a broad magnitude range. Two standard fields from the list of Landolt (1992), PG 1047+003 and SA 101, were observed to trace extinction and provide standard stars for the transformation. All these images were processed for bias and flat-fielding corrections with the standard procedures using the CCDPROC package in IRAF ${ }^{2}$.

Aperture photometry using the PHOT package inside DAOPHOT was carried out on the standard stars with the same aperture (35 pixels) for each filter. For stars in the field of NGC 3105 we performed photometry by point spread function (PSF) fitting using the DAOPHOT package (Stetson 1987) provided by IRAF. We used six-pixel apertures (similar to the full

\footnotetext{
2 IRAF is distributed by the National Optical Astronomy Observatories, which are operated by the Association of Universities for Research in Astronomy, Inc., under cooperative agreement with the National Sci-
} ence Foundation. 


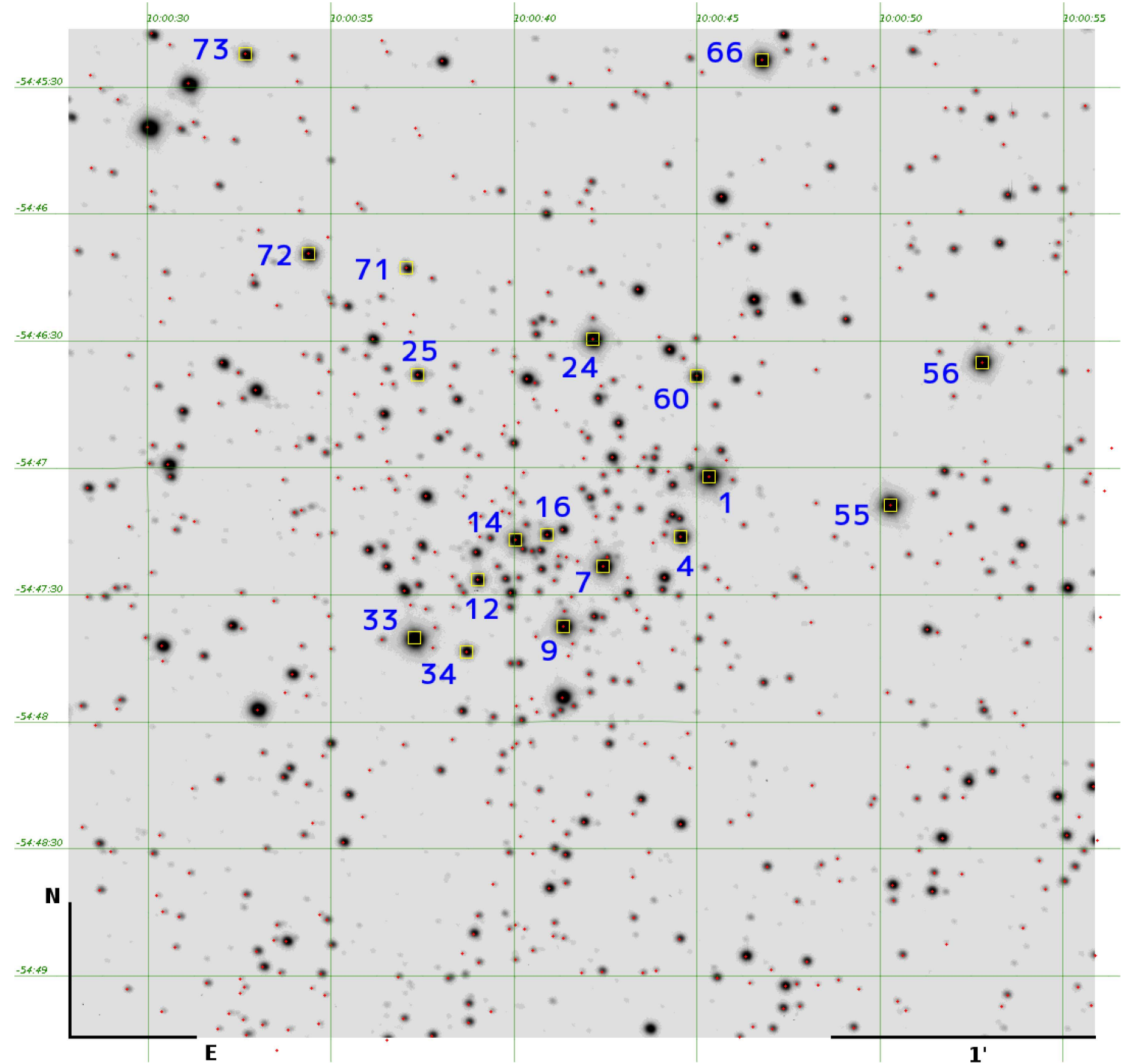

Fig. 1. Finding chart for the 607 stars with photometry in the field of $4.2^{\prime} \times 4.2^{\prime}$ centred on NGC 3105 (red dots). Yellow squares highlight the stars for which we have obtained spectra with the exception of stars 101 and 406, which fall outside the field of view covered by the photometry. The identification of each star corresponds to the WEBDA numbering for this cluster. The image is one of our $V$-band frames. North is up and east is right.

width at half maximum) for each image in all filters. To construct the PSF empirically, we selected bright stars (around 25) and discarded those with close companions or any other impediment. With the good PSF stars $(\approx 20)$, we determined an initial PSF by fitting the best function of the five options offered by the PSF routine inside DAOPHOT. We allowed the PSF to be variable (of order 2) across the frame because there may be systematic patterns of PSF variability with position on the chip. We then performed aperture correction for each frame in all filters.

Finally, we obtained the instrumental magnitudes for all stars. Using the standard stars in the Landolt fields, we carried out the atmospheric extinction correction and transformed the instrumental magnitudes (denoted with the subscript $i$ ) to the standard system with the PHOTCAL package inside IRAF. The equations used are as follows:

$$
\begin{aligned}
& U_{i}=(U-B)+(B-V)+V+(1.30 \pm 0.03) \\
& +(0.48 \pm 0.02) \cdot X_{U}-(0.046 \pm 0.007) \cdot(U-B)
\end{aligned}
$$

$$
\begin{aligned}
& B_{i}=(B-V)+V-(0.727 \pm 0.016) \\
& +(0.233 \pm 0.012) \cdot X_{B}-(0.038 \pm 0.003) \cdot(B-V) \\
& V_{i}=V-(0.83 \pm 0.02)+(0.114 \pm 0.016) \cdot X_{V} \\
& -(0.069 \pm 0.014) \cdot(B-V) \\
& R_{i}=V-(V-R)-(0.95 \pm 0.02) \\
& +(0.07 \pm 0.02) \cdot X_{R}-(0.016 \pm 0.014) \cdot(V-R)
\end{aligned}
$$

In total, we have photometry for 607 stars, which are displayed in Fig. 1 as red dots. In Table A.1 we list the identification number of each star $(I D)$; the equatorial coordinates (RA, DEC); the values of $V,(B-V),(U-B)$, and $(V-$ $R)$, together with their uncertainties; and the number of measurements $(N)$. The magnitudes and colours displayed in the table are the weighted arithmetic means (using the variances as weights) of all the individual measurements. The errors 
Table 2. Comparison of different photometries for NGC 3105 (this work - the literature), with $N$ being the number of stars in common.

\begin{tabular}{lccccccccc}
\hline \hline Reference & Phot. & $\Delta V$ & $N$ & $\Delta(B-V)$ & $N$ & $\Delta(U-B)$ & $N$ & $\Delta(V-R)$ & $N$ \\
\hline Lynga \& Wramdemark (1973) & $\mathrm{Pe}$ & $0.02 \pm 0.04$ & 9 & $0.01 \pm 0.07$ & 10 & $-0.07 \pm 0.07$ & 8 & - & - \\
Moffat \& Fitzgerald (1974) & $\mathrm{Pe}$ & $0.03 \pm 0.10$ & 12 & $-0.04 \pm 0.04$ & 12 & $-0.12 \pm 0.22$ & 9 & - \\
Fitzgerald et al. (1977) & $\mathrm{Pe}$ & $-0.00 \pm 0.10$ & 27 & $-0.05 \pm 0.06$ & 27 & $-0.01 \pm 0.14$ & 27 & - \\
Sagar et al. (2001) & $\mathrm{CCD}$ & $-0.13 \pm 0.14$ & 69 & $-0.07 \pm 0.11$ & 60 & - & - & $-0.14 \pm 0.09$ & - \\
\hline
\end{tabular}

are expressed in terms of the standard deviation around this mean.

When possible, we also show their 2MASS counterpart. The astrometric transformation, from pixel $(X, Y)$ to celestial coordinates (RA, DEC), was done in a first step using the XY2SKY task provided by WCSTOOLS (Mink 2002), and in a second pass with a subsequent correction employing as an astrometric reference 35 stars from the 2MASS catalogue, homogeneously distributed throughout the cluster field. The typical error of the astrometric fit, in both coordinates, is around 0 !' 3 .

As mentioned in Sect. 1 there are a few previous photometric studies for this cluster. We used the WEBDA database ${ }^{3}$ (Netopil et al. 2012), to download their data and compare their photometric values to ours. Moreover, we also included data from Lynga \& Wramdemark (1973) who obtained photoelectric magnitudes of stars in the field around the cluster (but not of the cluster itself). In Table 2 we list the differences (present work minus the literature data) and the number of stars in common $(N)$ with each work. We find no systematic deviations with respect to the photoelectric photometry, and a remarkable agreement with the values determined by Fitzgerald et al. (1977).

Following the method of Monguió et al. (2013), we estimate for our photometry a completeness of around $90 \%$ at these limiting magnitudes for each band: $U=21.3, B=20.7, V=19.3$, and $R=18.7$.

\section{2. $2 M A S S$ data}

We completed our dataset with $J H K_{\mathrm{S}}$ magnitudes from the 2MASS catalogue (Skrutskie et al. 2006). We cross-correlated the 2MASS catalogue with our photometric list of targets to find their infrared magnitudes. Only stars with good-quality photometry (i.e. without a " $U$ " photometric flag in the catalogue) were selected.

\subsection{Spectroscopy}

We took spectra for 20 stars by using three different spectrographs with several set-ups; 18 of the 20 stars covered by the photometry are indicated by yellow squares in Fig. 1. These objects were selected based on the literature and on their location in the 2MASS colour-magnitude diagram (CMD) in order to observe the brightest blue members as well as those evolved stars that have left the main sequence.

We collected spectra with the Cassegrain Spectrograph, mounted on the $1.9 \mathrm{~m}$ telescope at the South African Astronomical Observatory (SAAO). The spectra were taken in visitor mode from April 11 to 18, 2012. The spectral range and the resolution of the spectra depend on the diffraction grating used. We employed the gratings 7,8 , and 10 in order to observe the blue spectral region, the $\mathrm{H} \alpha$ line, and the $\mathrm{Ca}$ II triplet, respectively. Table 3 lists their characteristics. For wavelength calibration, we

\footnotetext{
3 Available at http://univie.ac.at/webda/
}

Table 3. Instrumental set-ups for the different spectrographs used.

\begin{tabular}{lccc}
\hline \hline Set-up & $\begin{array}{c}\text { Range } \\
(\AA)\end{array}$ & $\begin{array}{c}\text { Dispersion } \\
\left(\AA^{\circ} \text { pix }^{-1}\right)\end{array}$ & $S / N$ \\
\hline \multicolumn{4}{c}{ EFOSC2 } \\
\hline Grism 13 & $3685-9315$ & 2.77 & - \\
Grism 14 & $3750-5700$ & 0.93 & 75 \\
Grism 19 & 4235-4875 & 0.34 & 100 \\
\hline \multicolumn{4}{c}{ Cassegrain Spectrograph } \\
\hline Grating 7 & $3093-7105$ & 2.72 & 110 \\
Grating 8 & $6425-9279$ & 2.21 & 80 \\
Grating 10 & $8306-8927$ & 0.47 & 40 \\
\hline
\end{tabular}

used $\mathrm{CuAr}$ and $\mathrm{CuNe}$ arc spectra interspersed between the observations. The reduction was carried out with standard IRAF tools.

EFOSC2 is a versatile instrument that also works as a longslit spectrograph. We used grism 14 and the volume phase holographic (VPH) grism 19 to observe the blue spectral region with the highest resolution available. The grisms were used in combination with an off-centre slit to displace their nominal wavelength range to those shown in Table 3. The typical signalto-noise ratios $(\mathrm{S} / \mathrm{N})$ obtained for these spectra are listed in Table 3. The EFOSC2 spectra were reduced following standard procedures with the STARLINK software packages CCDPACK (Draper et al. 2011) and FIGARO (Shortridge et al. 2014). In addition, we obtained slitless spectroscopy of the field by combining the low-resolution grism 13 with the Gunn $r$-band filter. Two slitless images at different orientations were taken to minimise source overlapping.

Finally, we obtained high-resolution échelle spectra using the Fiber Extended Range Optical Spectrograph (FEROS) mounted on the ESO/MPG $2.2 \mathrm{~m}$ telescope at the La Silla Observatory in Chile. FEROS (Kaufer et al. 1999) covers the wavelength range from 3500 to $9200 \AA$, providing a resolving power of $R=48000$. This spectral region is covered in 39 orders, with small gaps between the orders appearing only at the longest wavelengths. The spectra were taken during two consecutive nights on May 29-30, 2015. The exposure times ranged from 2700 to 5400 s to achieve a typical $S / N \approx 70-80$. The spectroscopic reduction was performed using the FEROS-DRS pipeline based on MIDAS routines comprising the usual steps: bad pixel and cosmic correction, bias and dark current subtraction, removal of scattered light, optimum order extraction, flat-fielding, wavelength calibration using Th-Ar lamps exposures, and rectification and merging of the échelle orders.

\section{Results}

Throughout this paper we use the WEBDA numbering system to identify cluster stars observed spectroscopically. The designation in our photometry (ID) can be found in Tables A.1 and A.2. Table 4 lists both designations for stars with spectra. 
Table 4. Spectral types, spectrographs used, and estimates of cluster membership are shown for stars observed spectroscopically in the field of NGC 3105.

\begin{tabular}{|c|c|c|c|c|c|c|c|c|c|}
\hline \multicolumn{2}{|c|}{ Star } & \multirow[t]{2}{*}{ Spectral type } & \multicolumn{2}{|c|}{ EFOSC2 } & \multicolumn{3}{|c|}{ SAAO } & \multirow[t]{2}{*}{ FEROS } & \multirow[t]{2}{*}{ Membership } \\
\hline WEBDA & ID & & Gr. 14 & Gr. 19 & Gr. 7 & Gr. 8 & Gr. 10 & & \\
\hline 1 & 431 & $\mathrm{~A} 0 \mathrm{Ib}$ & $\bullet$ & $\bullet$ & $\bullet$ & $\bullet$ & & & $\mathrm{y}$ \\
\hline 4 & 418 & B2 IVe & $\bullet$ & $\bullet$ & & & & & $\mathrm{y}$ \\
\hline 7 & 358 & $\mathrm{~B} 1.5 \mathrm{Ve}$ & $\bullet$ & $\bullet$ & $\bullet$ & & $\bullet$ & & $\mathrm{y}$ \\
\hline 9 & 316 & K3 Ib & & & & & $\bullet$ & • & $\mathrm{y}$ \\
\hline 12 & 229 & $\mathrm{~B} 2 \mathrm{~V}$ & $\bullet$ & & & & & & $\mathrm{y}$ \\
\hline 14 & 267 & B3 III & $\bullet$ & $\bullet$ & $\bullet$ & $\bullet$ & & & $\mathrm{y}$ \\
\hline 16 & 298 & $\mathrm{~B} 2.5 \mathrm{~V}$ & • & & & & & & $\mathrm{y}$ \\
\hline 24 & 348 & $\mathrm{~K} 3 \mathrm{Ib}+\mathrm{B} 2 \mathrm{~V}$ : & - & & & & $\bullet$ & • & $\mathrm{y}$ \\
\hline 25 & 191 & B2 V & $\bullet$ & & & & & & $\mathrm{y}$ \\
\hline 33 & - & M1 Iab & & & & & $\bullet$ & & $\mathrm{y}$ \\
\hline 34 & 221 & B2.5 V & $\bullet$ & & & & & & $\mathrm{y}$ \\
\hline 55 & 503 & F9 Ib & & & & & • & • & y \\
\hline 56 & 548 & A0 Ib & $\bullet$ & $\bullet$ & $\bullet$ & & $\bullet$ & & $\mathrm{y}$ \\
\hline 60 & 426 & B2 IVe? & & - & & & & & $\mathrm{y}$ \\
\hline 66 & 459 & K5 Ib & & & & & $\bullet$ & $\bullet$ & $\mathrm{y}$ \\
\hline 71 & 184 & B2 IV & $\bullet$ & & & & & & $\mathrm{y}$ \\
\hline 72 & 121 & $\mathrm{~B} 1.5 \mathrm{Ve}$ & & $\bullet$ & $\bullet$ & & & & $\mathrm{y}$ \\
\hline 73 & 181 & M2 III & & & & $\bullet$ & $\bullet$ & $\bullet$ & $\mathrm{n}$ \\
\hline 101 & - & $\mathrm{B} 2 \mathrm{Ve}$ & • & & & & & & y? \\
\hline 406 & - & M6 Ib & & & & & & - & y? \\
\hline
\end{tabular}

Notes. For a clear identification of every star both numbering systems, WEBDA and ours (ID), are shown.

\subsection{Spectral classification}

We obtained spectra for the brightest stars in the cluster. In total, we collected 41 spectra for 20 different stars (see Table 4 for spectrograph set-ups and spectral types assigned). Taking into account all the configurations used, we covered at different resolutions the spectral range of 3100-9200 $\AA$, which includes the main spectral classification regions for both blue and red stars. We estimate for our classification a typical uncertainty of around one spectral subtype.

\subsubsection{Blue stars}

We took spectra of the blue stars in the field of NGC 3105 to study the upper main sequence and find the spectral type at which the main sequence turnoff (MSTO) point is seen on the CMD. We followed classical criteria of classification in the optical wavelength range (4000-5000 $)$, following Jaschek \& Jaschek (1987) and Gray \& Corbally (2009). From 13 low- and intermediate-resolution spectra taken with EFOSC2 (Gr14 and Gr19) and the SAAO Cassegrain Spectrograph (Gr7) we found two A supergiants (A0 Ib) and 11 B-type stars.

With respect to the A supergiants, around spectral type A0 the $\mathrm{Ca}$ II K-line is a notable feature, while the profile of the Balmer lines is the primary luminosity criterion: the narrower their wings, the higher the luminosity. The Fe II line at $4233 \AA$, the blends of Fe II and Ti II at 4172-8 $\AA$, and the Si II $24128-30$ doublet are enhanced in the supergiants, but at our resolution these features are not clearly separated, although visible in the spectra.

Regarding the B-type stars, as we move toward later spectral types the ratio $\mathrm{Mg}$ II $\lambda 4481 / \mathrm{He}_{\text {I }} \lambda 4471$ and the strength of the Ca II $K$ line increase as the He I lines weaken. Our sample consists of early $B$ stars with spectral types between B1.5 and B3. The line ratios used in this range to assign spectral types are $\mathrm{Si}$ II $24128-30$ /
Si III $\lambda 4553$, Si II $\lambda 4128-30 / \mathrm{He}_{\text {I }} \lambda 4121, \quad$ N II $\lambda 3995 / \mathrm{He}_{\text {I }} \lambda 4009$, and He I $_{4} 4121 / \mathrm{He}_{\mathrm{I}} \lambda 4144$ (Walborn \& Fitzpatrick 1990). A sample of the brightest blue stars, including the A0 supergiants, is shown in Fig. 2.

The earliest spectral types for MS stars, with no emission lines, were found around B2-B2.5. In addition, five of the B-type stars observed, namely stars 4, 7, 60, 72, and 101, are emissionline stars $(\mathrm{Be})$ with spectral types B1.5-B2. Stars 72 and 7 stand out because of their strong emission, showing $(\mathrm{H} \alpha)$ equivalent widths (EW) of $-73 \AA$ and $-67 \AA$, respectively. These are extreme values for classical Be stars. Only two objects among the more than 50 Be stars monitored by Barnsley \& Steele (2013) have ever shown comparable values. In addition, six other Be stars have been identified in the slitless fields, namely stars 40, 78, 84, 92, 213 (with no 2MASS counterpart), and 389. Among all these Be stars, star 101 is the only one far away from the cluster core; for this reason its membership is doubtful. However, early-type stars like this are not abundant in the sky and, in addition, it presents a spectral type similar to that of the other brightest blue members. This might suggest some sort of relationship with the cluster despite its distance to the core. In Fig. 3 we show $\mathrm{H} \alpha, \mathrm{H} \beta$, and $\mathrm{H} \gamma$ line profiles for all the Be stars with long-slit spectra.

\subsubsection{Red stars}

We obtained spectra for seven evolved stars in the cluster field. We classified them by comparison with the high-resolution standards from the atlas of Marrese et al. (2003) focusing on the near-infrared wavelength range around the Ca II triplet (8480$8750 \AA$ ). For this, we employed the spectra taken with the SAAO Cassegrain Spectrograph (Gr10) and FEROS, after degrading the latter to the resolution of the standards $(R \approx 20000)$. The triplet weakens toward later spectral types and lower luminosity classes (Jaschek \& Jaschek 1987), but many other classification criteria are available in this range. Many features 


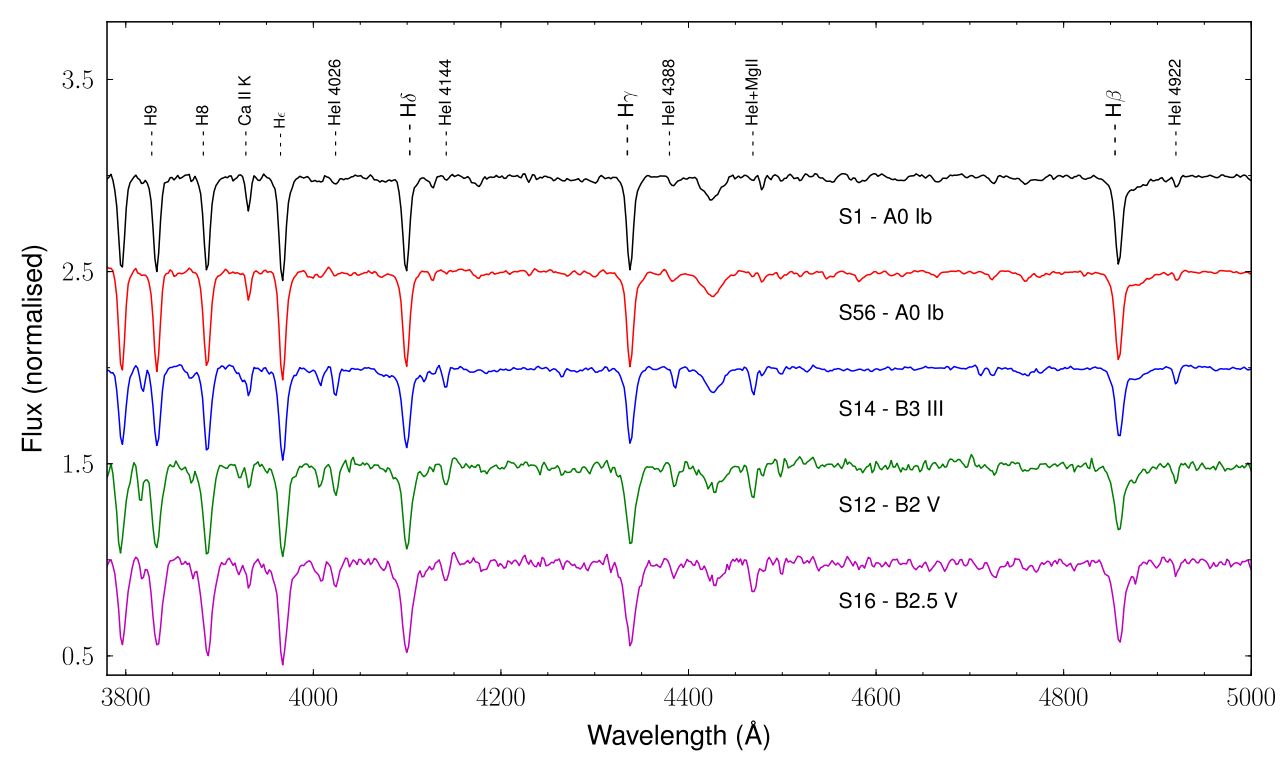

Fig. 2. Spectra of the brightest blue stars in the classical spectral classification region. These spectra have been obtained with grism 14 on EFOSC2. The most important lines are marked.

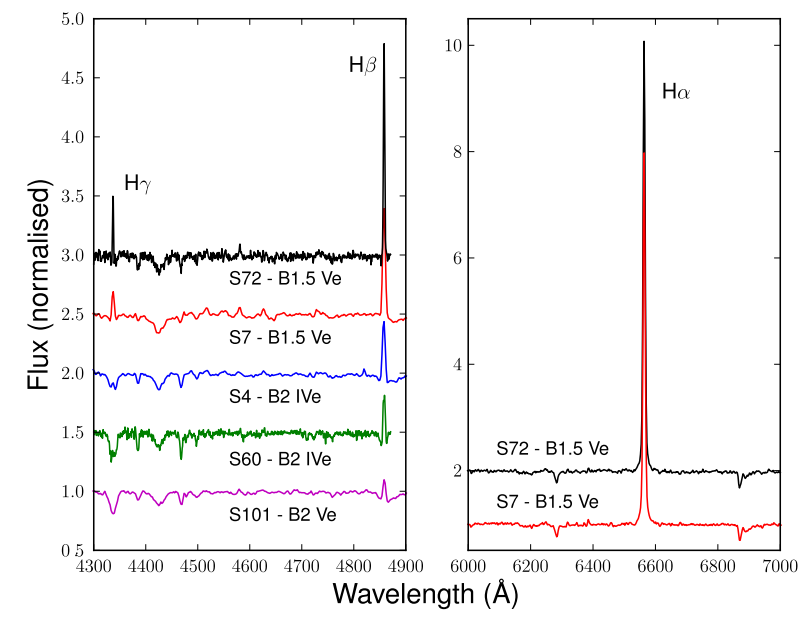

Fig. 3. Spectra of the Be stars in the field of NGC 3105. Left: EFOSC2 spectra around $\mathrm{H} \beta$ and $\mathrm{H} \gamma$ taken with grism 14 (for stars 4, 7, and 101) and 19 (60 and 72). Right: strong $\mathrm{H} \alpha$ emission in stars 7 and 72. These spectra were taken with grating 7 on the SAAO Cassegrain Spectrograph.

of Fe I (i.e. lines at 8514,8621 , and $8688 \AA$ ) and Ti I $(8518 \AA$ ) become stronger with later spectral types (Carquillat et al. 1997). In addition, we find very useful as classification criteria the ratios Ti I $\lambda 8518 / \mathrm{Fe}_{\text {I }} \lambda 8514$ and $\mathrm{Ti}$ I $\lambda 8734 / \mathrm{Mg}$ I $\lambda 8736$, which become higher with increasing spectral type.

We found one yellow supergiant (star 55-F8 Ib), three Ksupergiants $(9,24$, and 66) and three M-(super)giants (33, 73, and 406). Star 24 presents a composite spectrum, as first noted by Fitzgerald et al. (1977), consisting of an early B star and a cool supergiant. The hot companion is only detected by the presence of the Balmer lines in the blue region since the rest of the spectrum is dominated by the K-supergiant. The B-type component cannot thus be classified with accuracy. Figure 4 displays the spectra of evolved members belonging to the cluster. Star 73 is not included since its radial velocity (RV) is not compatible with that of the cluster (for discussion, see Sect. 3.5).

\subsection{Observational HR diagram: cluster reddening, distance and age}

We start the photometric analysis by plotting the $V /(B-V)$ colour-magnitude diagram (CMD) for all the stars in the field (see Fig. 5). We have highlighted spectroscopically observed stars, which clearly follow a sequence. Given the spectral types of the earliest stars observed and the completeness levels calculated above, we estimate our photometry to be complete down to the typical magnitude of $\approx \mathrm{A} 3 \mathrm{~V}$ stars.

In order to complement our photometry we used the 2MASS photometry. In Fig. 6, we also show the $K_{\mathrm{S}} /\left(J-K_{\mathrm{S}}\right)$ CMD from the 2MASS data for stars located up to $5^{\prime}$ from the nominal centre. Because of its high distance modulus, the top of the MS is found only one and a half magnitudes above the completeness limit in the $K_{\mathrm{S}}$ band $(\sim 14.3)$. Thus, the cluster is too faint for performing a complete analysis with the 2MASS data, analogous to that carried out with the optical photometry. However, this diagram (Fig. 6) shows the position of star 406, a possible new RSG (whose cluster memberhip has not been claimed so far), not covered by the optical photometry.

\subsubsection{Reddening}

Firstly, we selected the B-type stars spectroscopically identified in that sequence (Fig. 5) without emission lines or red companions. For these stars, six in total, we determined their individual reddening by comparing the observed $(B-V)$ colours with the $(B-V)_{0}$ corresponding to their spectral types (Table 5). We made a first estimate of the cluster reddening by taking an average of the values for these stars. We obtained a colour excess $E(B-V)=1.03 \pm 0.03$. The low dispersion suggests an absence of strong differential reddening that allows a meaningful determination of the average. In order to estimate whether the extinction law is standard in this direction, we also calculated for these six stars the ratio $X=E(U-B) / E(B-V)$. We find an average $X=0.76 \pm 0.11$, compatible with the standard value of 0.72 (Johnson \& Morgan 1953). In both cases the intrinsic colours were adopted from Fitzgerald (1970) and the errors show the dispersion between different stars. 


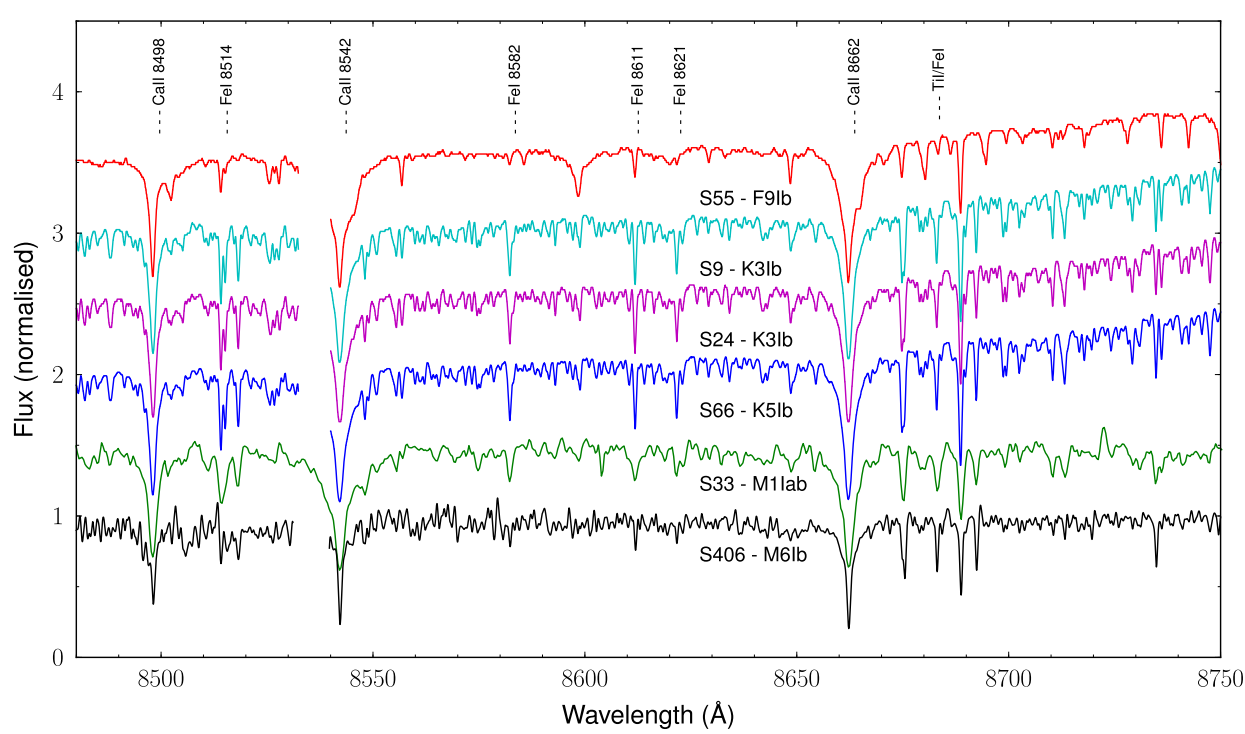

Fig. 4. Spectra of cool supergiant members around the Ca II triplet. All the spectra were taken with FEROS (there is a gap between orders 37 and 38 around $8535 \AA$ ), except that of star 33, which was taken with grating 10 on the SAAO Cassegrain Spectrograph.

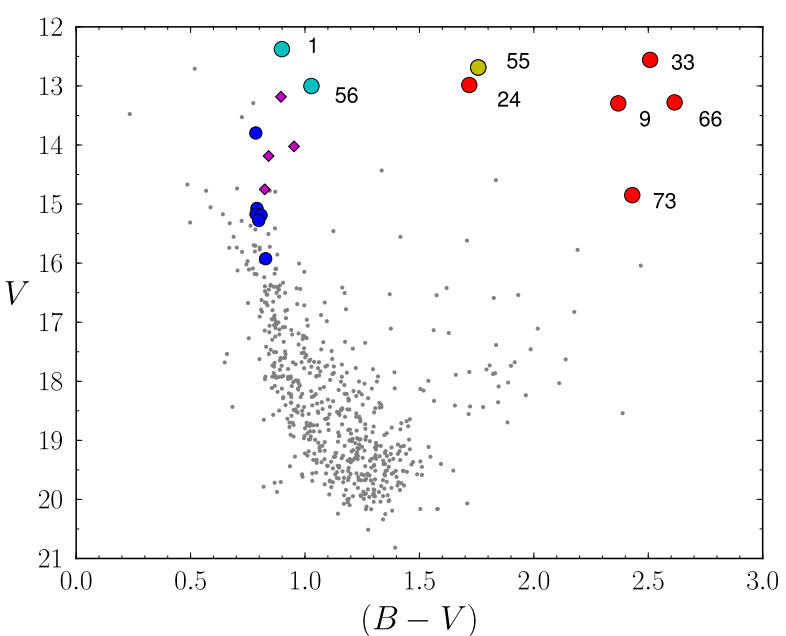

Fig. 5. $V /(B-V)$ diagram for all stars in the field of NGC 3105. Photometric data appear as grey dots. Stars for which we also have spectra are represented by small blue circles (upper main sequence B-type stars), magenta diamonds (Be stars), and filled circles (supergiant stars; colour indicates spectral type: cyan for BSGs, yellow for YSG, and red for RSGs).

\subsubsection{Cluster membership and fitting the ZAMS}

Since blue members in stellar clusters are easy to distinguish from the field population, and are thus good tracers of the cluster extent, we looked for them in the range from the earliest objects found with spectral types B2-2.5 down to the spectral type B9, for which we have completeness in our photometry as mentioned above (Sect. 3.2). Since the extinction is close to standard, we can use the classical $Q$ parameter (Johnson \& Morgan $1953)$, defined as $Q=(U-B)-X(B-V)$. This parameter, which is corrected from interstellar reddening, allows the efficient separation of blue stars from the rest and assigns reliable photometric spectral types. From the photometry and the average $X$ ratio found in the previous subsection we calculated the value of $Q$ for the totality of objects that we have. In order to find stars intrinsically blue along the cluster field, we chose stars with $-0.74<Q<-0.06$, as described in Johnson \& Morgan (1953).

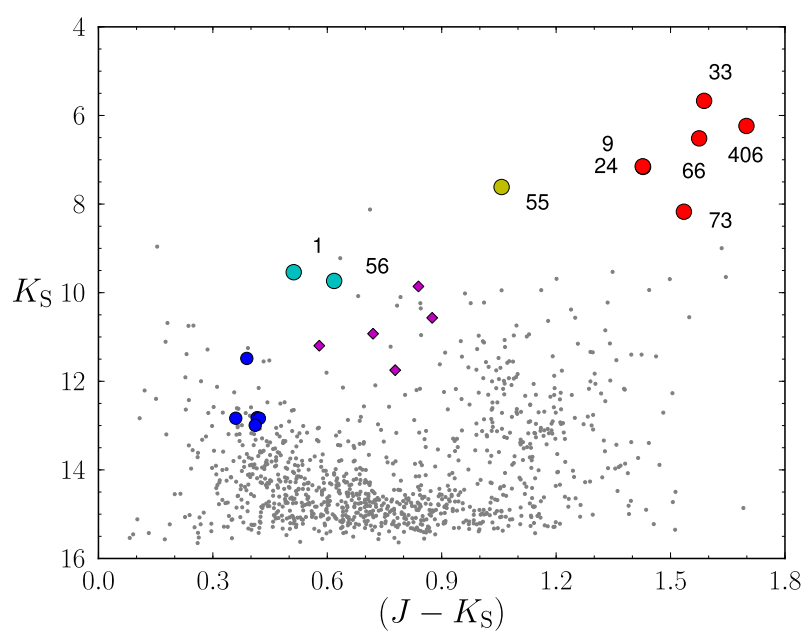

Fig. 6. $K_{\mathrm{S}} /\left(J-K_{\mathrm{S}}\right)$ diagram for all stars with good 2 MASS data in the field of NGC 3105. Symbols and colours are as in Fig. 5. Stars 9 and 24 occupy the same position in the diagram. Also shown is the location of S406, a likely new cluster RSG not covered by the optical photometry.

Then, we assigned photometric spectral types to these stars, and selected as likely members those whose photometric spectral types are in fair agreement with their expected location on the CMD (Fig. 5). For the early-type stars with spectra, the difference between the spectroscopic and photometric spectral types is typically only one subtype. Finally, we find 126 likely members, whose individual photometric reddening can be estimated using this expression (Johnson 1958):

$E(B-V)=(B-V)-0.332 Q$

For these stars, the average reddening is $E(B-V)=1.01 \pm$ 0.04 , fully compatible with that obtained for the limited sample of objects with spectra using the colour calibration. In Table A.2, we list the likely members together with the $Q$ index, photometric spectral type, and reddening estimated in this section. In addition, the last column of Table 4 summarises the cluster membership for all stars observed spectroscopically. Most of them are likely blue members, as just discussed. The 
Table 5. Colour excesses and absolute magnitudes in the $V$ band for B-type stars in the field of NGC 3105 .

\begin{tabular}{lccccccccc}
\hline \hline NGC 3105 & Sp T & $(B-V)$ & $(U-B)$ & $(B-V)_{0}$ & $(U-B)_{0}$ & $E(B-V)$ & $E(U-B)$ & $X$ & $M_{V}$ \\
\hline 12 & B2 V & 0.790 & -0.017 & -0.24 & -0.81 & 1.030 & 0.793 & 0.770 & -2.31 \\
14 & B3 III & 0.785 & -0.004 & -0.20 & -0.73 & 0.985 & 0.726 & 0.737 & -3.55 \\
16 & B2.5 V & 0.808 & 0.060 & -0.22 & -0.72 & 1.028 & 0.780 & 0.759 & -2.19 \\
25 & B2 V & 0.786 & -0.020 & -0.24 & -0.81 & 1.026 & 0.790 & 0.770 & -2.20 \\
34 & B2.5 V & 0.798 & -0.003 & -0.22 & -0.72 & 1.018 & 0.717 & 0.704 & -2.12 \\
71 & B2 IV & 0.828 & -0.008 & -0.24 & -0.86 & 1.068 & 0.852 & 0.798 & -1.60 \\
\hline
\end{tabular}

Notes. Intrinsic colours are adopted from Fitzgerald (1970).

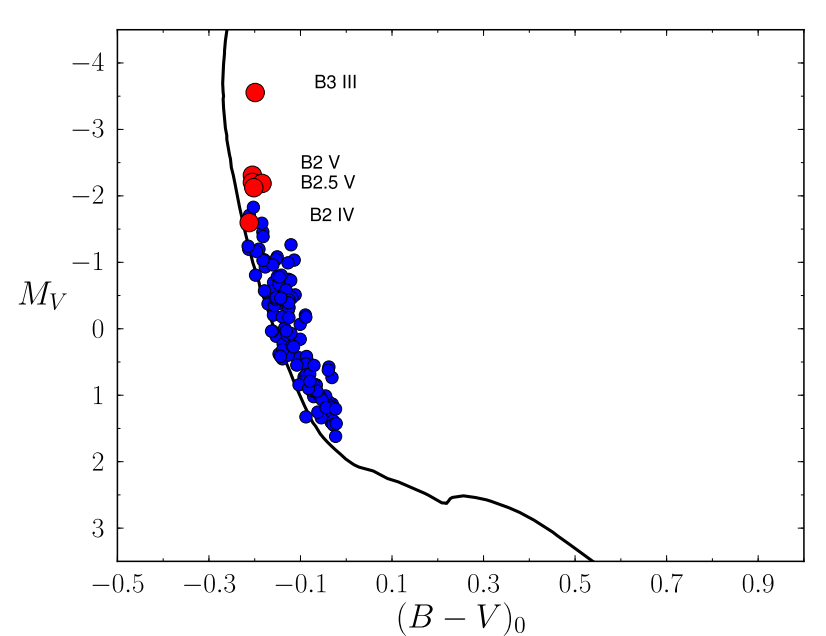

Fig. 7. $M_{V} /(B-V)_{0}$ diagram for likely members of NGC 3105 . Stars observed spectroscopically are represented in red. Spectral types for the brightest stars are marked. Blue circles are the rest of the early members found photometrically. The black line shows, as a ZAMS, a PARSEC isochrone of $10 \mathrm{Ma}$ computed at the cluster metallicity. The best-fitting yields a distance modulus of $\mu=14.3 \pm 0.2$.

criteria for selecting red members are based on the position on CMD (Sect. 3.2.3) and on the analysis of radial velocities (Sect. 3.5).

Once we know the individual reddening for likely blue members, we carefully performed a visual fit of these dereddened values with the observational zero age main sequence (ZAMS). Classical ZAMSs (such as that from Aller et al. 1982) have been calibrated with bright stars in the solar neighbourhood, which are easily observable and typically have solar abundances. Since this cluster exhibits a lower metallicity, it is necessary to employ a more appropriate ZAMS. To this end, we computed a PARSEC isochrone with the cluster metallicity, but with an age young enough (i.e. $10 \mathrm{Ma}$ ) compared to that of the cluster itself. From the ZAMS fitting (see Fig. 7) we obtained the distance modulus, $\mu=V_{0}-M_{V}=14.3 \pm 0.2$, which is equivalent to a distance $d=7.2 \pm 0.7 \mathrm{kpc}$. The error reflects the uncertainty when visually fitting the ZAMS as a lower envelope. This distance places the cluster with respect to the Galactic centre, taking as solar reference $R_{\odot}=8.3 \mathrm{kpc}$, at $R_{\mathrm{GC}}=10.0 \pm 0.9 \mathrm{kpc}$.

Finally, we took advantage of the combination of photometry with spectroscopy to check the validity of our result. In this way, the $M_{V}$ resulting for stars with spectra are compatible with those expected according to their spectral types.

\subsubsection{Fitting isochrones}

We employed the isochrone fitting method to determine the age of the cluster. Taking into account the distance modulus just

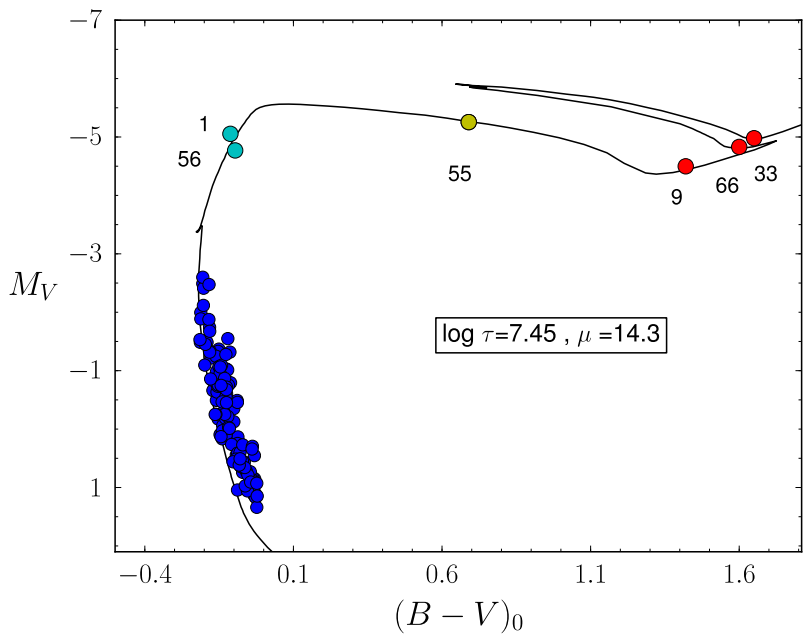

Fig. 8. $M_{V} /(B-V)_{0}$ diagram for likely members of NGC 3105 . Symbols and colours are as in Fig. 5. The best-fitting isochrone is the black line.

estimated, we initially plotted the $M_{V} /(B-V)_{0}$ diagram and then, on it, we fitted by eye PARSEC isochrones computed at the cluster metallicity (Bressan et al. 2012). The metallicity obtained in this work (see Sect. 3.6.2) in terms of iron abundance is $[\mathrm{Fe} / \mathrm{H}]=-0.29$. We converted this value into $Z$ by using the approximation $[\mathrm{M} / \mathrm{H}]=\log \left(Z / Z_{\odot}\right)$, with $Z_{\odot}=0.0152$ for PARSEC tracks.

Figure 8 shows the optical dereddened CMD, $M_{V} /(B-V)_{0}$, for likely members and the best-fitting isochrones. The dereddening of the blue members has been described before. For all supergiants we followed the procedure described by Fernie (1963). Only single stars without emission lines are included. Although we do not provide photometry for S33, we use the value given by Fitzgerald et al. (1977) taking into account that there is no offset between the two photometries (Table 2). When fitting the isochrones, it is important to pay special attention to the position of the evolved members, from which we infer the cluster age. The best-fitting PARSEC isochrone gives $\log \tau=7.45 \pm$ 0.10 , where the error represents the typical range of isochrones which give a good fit. At this age, the mass of the RSGs would correspond to $9-10 M_{\odot}$, somewhat smaller than that expected from previous works.

\subsection{Cluster geometry}

From the sky density distribution of the 126 likely members the centre and size of the cluster are derived as described in Monguió et al. (2017). For this purpose the observed sky field has been divided symmetrically into a grid of points spaced every $5^{\prime \prime}$. The sky density has been computed as the number of stars located closer than 0.5 from each point of this grid. The cluster is enhanced from the background and the overdensity at 


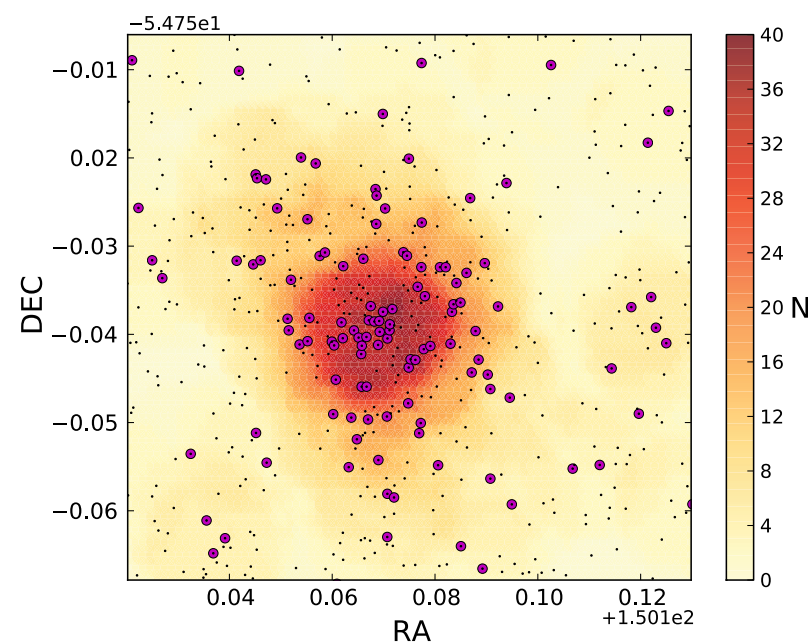

Fig. 9. Sky distribution of the likely members of NGC 3105 (magenta circles) and the rest of the observed stars (black dots) in the field covered by our photometry. Density is computed as the number of stars closer than 0 '.5 to each point. The overdensity corresponding to the cluster core clearly stands out in the figure.

its central part is clearly detected (see Fig. 9). Then, we fit this density with a 2D Gaussian such that

$n=n_{0}+n_{\max } \cdot \mathrm{e}^{-\left(A\left(\alpha-\alpha_{0}\right)^{2}+2 B\left(\alpha-\alpha_{0}\right)\left(\delta-\delta_{0}\right)+C\left(\delta-\delta_{0}\right)^{2}\right)}$

with

$A=\frac{\cos ^{2} \theta}{2 \sigma_{\alpha}^{2}}+\frac{\sin ^{2} \theta}{2 \sigma_{\delta}^{2}}$,

$B=\frac{-\sin (2 \theta)}{4 \sigma_{\alpha}^{2}}+\frac{\sin (2 \theta)}{4 \sigma_{\delta}^{2}}$,

$C=\frac{\sin ^{2} \theta}{2 \sigma_{\alpha}^{2}}+\frac{\cos ^{2} \theta}{2 \sigma_{\delta}^{2}}$,

where there are seven parameters to fit: $n_{0}$ is the offset due to the zero point, $n_{\max }$ is the amplitude of the Gaussian, $\alpha_{0}$ and $\delta_{0}$ are the equatorial coordinates of the cluster centre, $\sigma_{\alpha}$ and $\sigma_{\delta}$ are the standard deviation corresponding to each coordinate, and $\theta$ is the position angle of the ellipse with respect to the vertical. The results, obtained through a least-squares fit, are listed in Table 6.

The cluster extends across the sky projecting an ellipse whose eccentricity is given by the expression

$\epsilon=\sqrt{1-\frac{\left(\sigma_{\alpha} \cos \delta_{0}\right)^{2}}{\sigma_{\delta}^{2}}}=0.51$.

Finally, we can define the radius of the cluster $\left(r_{\mathrm{cl}}\right)$ as the distance from its centre at which almost all the cluster members are contained (within $3 \sigma$ ):

$r_{\mathrm{cl}}=3 \sqrt{\left(\sigma_{\alpha} \cos \delta_{0}\right)^{2}+\sigma_{\delta}^{2}}$.

According to this definition, and taking into account the uncertainties in each of the parameters, we estimate a cluster radius, $r_{\mathrm{cl}}=2: 7 \pm 0 \prime 6$, that at the distance of the cluster corresponds with a physical size of $5.8 \pm 1.7 \mathrm{pc}$. This result is consistent with the position of stars 66 and 406 . Among those members confirmed via radial velocity, the two stars are the most distant objects from the cluster centre, located at around $2^{\prime}$, within the
Table 6. Parameters obtained for the cluster geometry.

\begin{tabular}{|c|c|}
\hline Parameter & Value \\
\hline$n_{0}$ & $2.286 \pm 0.009$ stars $\operatorname{arcmin}^{-2}$ \\
\hline$n_{\max }$ & $35.80 \pm 0.19$ stars $\operatorname{arcmin}^{-2}$ \\
\hline$\alpha_{0}$ & $150.17020 \pm 0.00007^{\circ}$ \\
\hline$\delta_{0}$ & $-54.78922 \pm 0.00005^{\circ}$ \\
\hline$\sigma_{\alpha}$ & $0.01397 \pm 0.00008^{\circ}$ \\
\hline$\sigma_{\delta}$ & $0.00915 \pm 0.00005^{\circ}$ \\
\hline$\theta$ & $4.8 \pm 0.5^{\circ}$ \\
\hline
\end{tabular}

radius found in this work. Star 101 lies in the cluster surroundings, at $5^{\prime}$, beyond the cluster radius. This implies that this star either is not a cluster member or is placed in the cluster outer halo.

\subsection{Cluster mass}

Once the size of the cluster is fixed, we continue our analysis by estimating its mass using the initial mass function (IMF). For this purpose we employed the multiple-part power law IMF defined by Kroupa (2001).

$\xi(M)=\xi_{0} \cdot M^{-\alpha_{i}}\left\{\begin{array}{ll}\alpha_{1}=1.3, & 0.08 \leq M / M_{\odot}<0.50 \\ \alpha_{2}=2.3, & 0.50 \leq M / M_{\odot}\end{array}\right.$,

where $\xi_{0}$ is the constant which sets the local stellar density.

By integrating the IMF over a mass range between $M_{1}$ and $M_{2}$, the number of stars $(N)$ expected in this interval is given by

$$
\begin{aligned}
& N=\int_{M_{1}}^{M_{2}} \xi(M) \mathrm{d} M=\xi_{0} \int_{M_{1}}^{M_{2}} M^{-\alpha_{i}} \mathrm{~d} M \\
& \Rightarrow \quad \xi_{0}=\frac{N}{\int_{M_{1}}^{M_{2}} M^{-\alpha_{i}} \mathrm{~d} M}
\end{aligned}
$$

Firstly, we set the free parameter of the $\operatorname{IMF}\left(\xi_{0}\right)$ by counting the stars within a certain mass range. We chose $B$ stars ranging from spectral type B2 V (which corresponds to the spectral type of the earliest members) to $\mathrm{B} 9 \mathrm{~V}$, where the separation from the field population is very efficient and completeness is ensured (Sect. 3.2). According to the calibration of Straižys (1992), this range of spectral types covers stars between 2.6 and $9.8 M_{\odot}$. These stars, all inside the cluster radius, are the 126 likely members, previously selected in Sect. 3.2.2.

Once the value of $\xi_{0}$ is known ( $\left.\xi_{0} \approx 707\right)$, the total mass of the cluster can be estimated using the following expression:

$M=\int_{M_{1}}^{M_{2}} M \xi(M) \mathrm{d} M=\xi_{0} \int_{M_{1}}^{M_{2}} M^{1-\alpha_{i}} \mathrm{~d} M$.

Then, integrating between 0.08 and $9.5 M_{\odot}$ (the mass of the evolved stars according to the isochrone) we obtain the present mass, whereas integrating up to $150 M_{\odot}$ (as a classical estimate of the maximum mass for a $\operatorname{star}^{4}$ ) we determine the initial mass. For NGC 3105 we find $\approx 2300$ and $\approx 3000 M_{\odot}$, respectively. Finally, according to Tadross (2005) we correct these values for unresolved binarity by assuming an average mass ratio of binary systems of 1.3 (Allen 1973) and a binary frequency of $50 \%$ (Jaschek \& Gómez 1970). Kouwenhoven et al.

\footnotetext{
4 Crowther et al. (2010) raise this value above $300 M_{\odot}$; however, this number, if confirmed as the actual initial mass of a single star or as the resulting mass of a stellar merger, hardly affects the cluster mass.
} 
Table 7. Log of EFOSC2 spectra and stellar atmospheric parameters for blue stars with no emission lines, and the stellar atmospheric parameters derived from them.

\begin{tabular}{lccccccc}
\hline \hline Star & Sp T & Grism & $t_{\exp }(\mathrm{s})$ & $S / N$ & $v \sin i\left(\mathrm{~km} \mathrm{~s}^{-1}\right)$ & $T_{\text {eff }}(\mathrm{K})$ & $\log g$ \\
\hline 1 & A0 Ib & 19 & 900 & 130 & 90 & $12000 \pm 1000$ & $2.6 \pm 0.1$ \\
12 & B2 V & 14 & 1800 & 60 & 110 & $18000 \pm 2200$ & $3.8 \pm 0.2$ \\
14 & B3 III & 19 & 1800 & 90 & 90 & $17000 \pm 1300$ & $3.1 \pm 0.2$ \\
16 & B2.5 V & 14 & 1800 & 160 & 230 & $17000 \pm 1000$ & $4.1 \pm 0.1$ \\
25 & B2 V & 14 & 1800 & 60 & 230 & $18000 \pm 1000$ & $4.1 \pm 0.1$ \\
34 & B2.5 V & 14 & 1800 & 60 & 210 & $16000 \pm 1000$ & $3.8 \pm 0.1$ \\
56 & A0 Ib & 19 & 1200 & 120 & 70 & $12000 \pm 1000$ & $2.6 \pm 0.1$ \\
71 & B2 IV & 14 & 1800 & 50 & 230 & $20000 \pm 1600$ & $4.1 \pm 0.2$ \\
\hline
\end{tabular}

Table 8. Log of FEROS spectra and stellar atmospheric parameters for the cool stars derived from them.

\begin{tabular}{lccccccccc}
\hline \hline Star & Sp T & $t_{\text {exp }}(\mathrm{s})$ & $S / N$ & $v_{\text {rad }}\left(\mathrm{km} \mathrm{s}^{-1}\right)$ & $v \sin i\left(\mathrm{~km} \mathrm{~s}^{-1}\right)$ & $\zeta\left(\mathrm{km} \mathrm{s}^{-1}\right)$ & $T_{\text {eff }}(\mathrm{K})$ & $\log g$ & {$[\mathrm{Fe} / \mathrm{H}]$} \\
\hline \multicolumn{7}{c}{} & \multicolumn{7}{c}{ Members } \\
\hline 9 & K3 Ib & 3600 & 80 & $47.16 \pm 0.03$ & $6.6 \pm 1.4$ & $1.56 \pm 0.90$ & $4038 \pm 134$ & $0.78 \pm 0.39$ & $-0.47 \pm 0.18$ \\
$24^{*}$ & $\mathrm{~K} 3 \mathrm{Ib}$ & 3600 & 80 & $40.60 \pm 0.04$ & $7.4 \pm 1.1$ & $7.50 \pm 0.25$ & $4285 \pm 80$ & $1.42 \pm 0.22$ & $-0.20 \pm 0.11$ \\
55 & F9 Ib & 3600 & 70 & $45.48 \pm 0.07$ & $7.0 \pm 1.6$ & $12.61 \pm 1.11$ & $5926 \pm 141$ & $1.57 \pm 0.30$ & $-0.24 \pm 0.10$ \\
66 & K5 Ib & 3600 & 100 & $46.82 \pm 0.03$ & $7.8 \pm 1.4$ & $3.66 \pm 1.05$ & $3930 \pm 147$ & $0.29 \pm 0.43$ & $-0.55 \pm 0.19$ \\
406 & M6 Ib & 2700 & 40 & $47.15 \pm 0.07$ & 9.7 & 1.90 & $3300 \pm 100$ & $0.00 \pm 0.50$ & -0.29 \\
\hline \multicolumn{7}{c}{} \\
\hline 73 & M2 III & 5400 & 60 & $59.38 \pm 0.04$ & 7.5 & 7.1 & $3600 \pm 100$ & 0.00 & -0.10 \\
\hline
\end{tabular}

Notes. ${ }^{*}$ This star exhibits a composite spectrum; the companion is a blue star (B2 V:).

(2005) found fractions of around $80 \%(\mathrm{~B} 0-\mathrm{B} 3)$ and $50 \%(\mathrm{~B} 4$ B9) among $B$ stars in the Sco OB2 association. Recently, Dunstall et al. (2015), in the 30 Dor star forming region, also estimated an observed mean binary fraction of around $25 \%$, but the implied underlying (true) value (according to their simulations) is $58 \%$, a fraction very similar to our assumption (50\%). We obtain a total present mass of $\approx 3200 M_{\odot}$, and a total initial mass around $4100 M_{\odot}$. Hence, we found that NGC 3105 is a moderately massive cluster.

\subsection{Rotational and radial velocities}

As mentioned before (Sect.2.3) we took low- and moderateresolution spectra for blue stars. This resolution is not as high as that necessary to separate different broadenings and we assumed that the whole broadening has a rotational origin. Starting from a first estimation of $v \sin i$ (around $50 \mathrm{~km} \mathrm{~s}^{-1}$, a value close to the resolution) an initial model capable of reproducing the spectrum was computed. In an iterative way, once we set the stellar parameters, we looked for a second estimate of $v \sin i$ by changing it until we found a new model which reproduced best the profiles. This process was repeated a few times until it did not change the rotational velocity. The values obtained for $v \sin i$, together with the atmospheric parameters, are listed in Table 7.

In contrast, for the six stars observed at high resolution with FEROS we computed $v \sin i$ by using the IACOB-BROAD code (Simón-Díaz \& Herrero 2014), based on the Fourier transform method, which separates rotation from other broadening mechanisms such as the macroturbulent velocity $(\zeta)$. We used six lines of $\mathrm{Fe}_{\mathrm{I}}$ and $\mathrm{Ni}$. The errors reflect the scatter between measurements, in terms of rms. The results are shown in Table 8 .

For these stars we also obtained RVs, referred to the heliocentric reference frame of rest, through Fourier cross-correlation by employing the software ISPEC (Blanco-Cuaresma et al. 2014), especially designed for the study of cool stars. The crosscorrelation is computed against a list of atomic line masks, carefully selected for the Gaia benchmark stars library pipeline, from asteroids observed with the NARVAL spectrograph.

Star 73 (See Table 8) presents a discrepant RV with respect to the rest, suggesting that it is a field star. This is confirmed by its low luminosity class. The remaining objects have very similar radial velocities; star 24 shows a RV slightly lower than the other supergiants, but this discrepancy is fully consistent with its binary nature. Also star 406 shows a RV compatible with other supergiants, but because of its uncertain nature (see discussion in Sect. 4.5) it has not been used to calculate the cluster average. Thus, we can use the other three stars (namely 9, 55, and 66) to estimate the RV for the cluster by averaging the individual values, obtaining a $v_{\text {rad }}=+46.9 \pm 0.9 \mathrm{~km} \mathrm{~s}^{-1}$, where the error is the dispersion between the values for the different stars.

\subsection{Stellar atmospheric parameters}

\subsubsection{Blue stars}

From low-medium resolution EFOSC2 spectra $(R \sim 1000)$ we computed stellar parameters for 12 blue stars. When possible we used the spectra taken with grism 19 since it provides a higher resolution than grism 14 .

We employed the technique described by Castro et al. (2012), also in Lefever (2007). The stellar atmospheric parameters were derived through an automatic $\chi^{2}$-based algorithm searching for the set of parameters that best reproduce the main strong lines observed in the range $\approx 4000-5000 \AA$. We used a grid of FASTWIND synthetic spectra (Simón-Díaz et al. 2011; Castro et al. 2012). The stellar atmosphere code FASTWIND (Santolaya-Rey et al. 1997; Puls et al. 2005) enables non-LTE calculations and assumes a spherical geometry and an explicit 
treatment of the stellar wind effects. The results are shown in Table 7. It should be noted that the emission present in the Be stars has prevented a good fit with the models, yielding unrealible parameters, such as gravities smaller than those computed for supergiants. For this reason, we ignore the results for these objects. Temperatures for non-emission $B$ stars are fully compatible with those from the calibration by Humphreys \& McElroy (1984). The temperatures for A0 supergiants are somewhat hotter than the calibration.

\subsubsection{Cool stars}

For the cool stars we derived stellar atmospheric parameters from their FEROS spectra. For the three K-supergiants and the yellow supergiant we followed the procedure described in Alonso-Santiago et al. (2017), but using different atmospheric models. In this work we took 1D LTE atmospheric models, specifically MARCS spherical models with $1 M_{\odot}$ (Gustafsson et al. 2008). We generated a grid of synthetic spectra by using the radiative transfer code SPECTRUM (Gray \& Corbally 1994). Although MARCS models are spherical, SPECTRUM treats them as if they are plane-parallel. Therefore, the plane parallel transfer treatment might produce a small inconsistency in the calculations of synthetic spectra based on MARCS atmospheric models. However, the study of Heiter \& Eriksson (2006) concluded that any difference introduced by the spherical models in a plane-parallel transport scheme is small. The microturbulent velocity $(\xi)$ was fixed according to the calibration given in Dutra-Ferreira et al. (2016). Effective temperature $\left(T_{\text {eff }}\right)$ ranges from $3300 \mathrm{~K}$ to $7500 \mathrm{~K}$ with a step of $100 \mathrm{~K}$ up to $4000 \mathrm{~K}$ and $250 \mathrm{~K}$ until $7500 \mathrm{~K}$, whereas surface gravity ( $\log g$ ) varies from -0.5 to 3.5 dex in 0.5 dex steps. Finally, in the case of metallicity (using $[\mathrm{Fe} / \mathrm{H}]$ as a proxy), the grid covers from -1.5 to +1.0 dex in 0.25 dex steps.

We employed a methodology to derive stellar atmospheric parameters based on the iron linelist provided by Genovali et al. (2013) since Fe lines are numerous and are very sensitive in cool stars. The linelist contains $\sim 230$ features for $\mathrm{Fe}_{\mathrm{I}}$ and $\sim 55$ for Fe II. Their atomic parameters were taken from the VALD database $^{5}$ (Piskunov et al. 1995; Kupka et al. 2000). For the Van der Waals damping data, we took the values given by the Anstee, Barklem, and O'Mara theory, when available in VALD (see Barklem et al. 2000).

As a starting point, we employed an updated version of the STEPAR code (Tabernero et al. 2018), adapted to the present problem, which uses stellar synthesis instead of an $E W$ method. As the optimisation method we used the Metropolis-Hastings algorithm. Our method simultaneously generates 48 Markov chains of 750 points each. It then performs a Bayesian parameter estimation by employing an implementation of the Goodman \& Weare Affine Invariant Markov chain Monte Carlo Ensemble sampler (Foreman-Mackey et al. 2013). As objective function we used a $\chi^{2}$ in order to fit the selected iron lines. We fixed the stellar rotation to that value previously derived, and the instrumental broadening to the resolution of the FEROS spectrograph. We left the macroturbulent broadening as a free parameter to absorb any residual broadening. An example of the spectral line fitting performed with our methodology is shown in Fig. 10.

In the case of the M-(super)giants, since their spectra are dominated by molecular bands which erode the continuum weakening or erasing other spectral features such as most

\footnotetext{
5 http://vald.astro.uu.se/
}

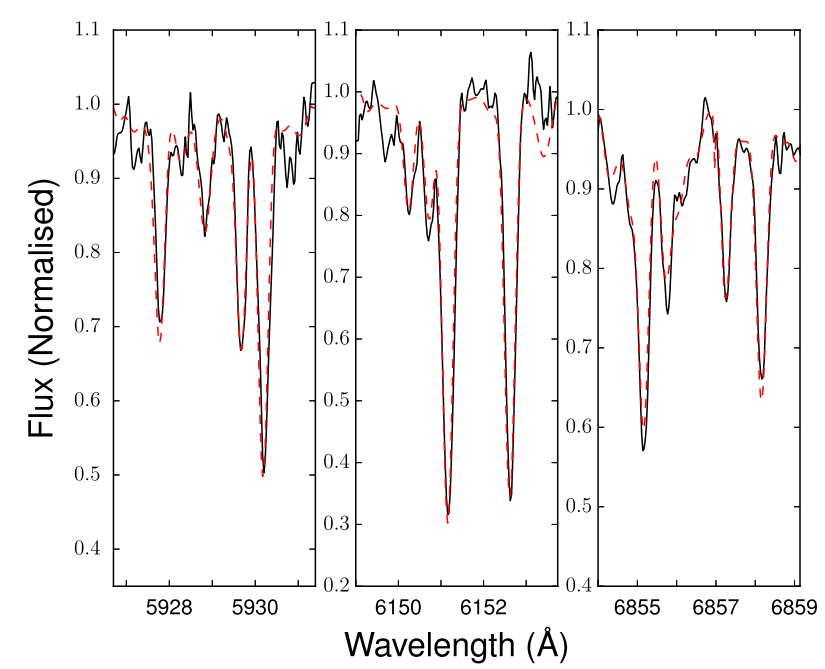

Fig. 10. Some examples of line fits in star 66. Three regions are shown around $\mathrm{Fe}_{\mathrm{I}} \lambda 5927.80$ (left), Fe II $\lambda 6149.25$ (centre), and Fe I $\lambda 6855.74$ $\mathrm{Fe}_{\mathrm{I}} 16858.16$ (right). The observed spectrum is the solid black line, whereas the synthetic one is the red dashed line.

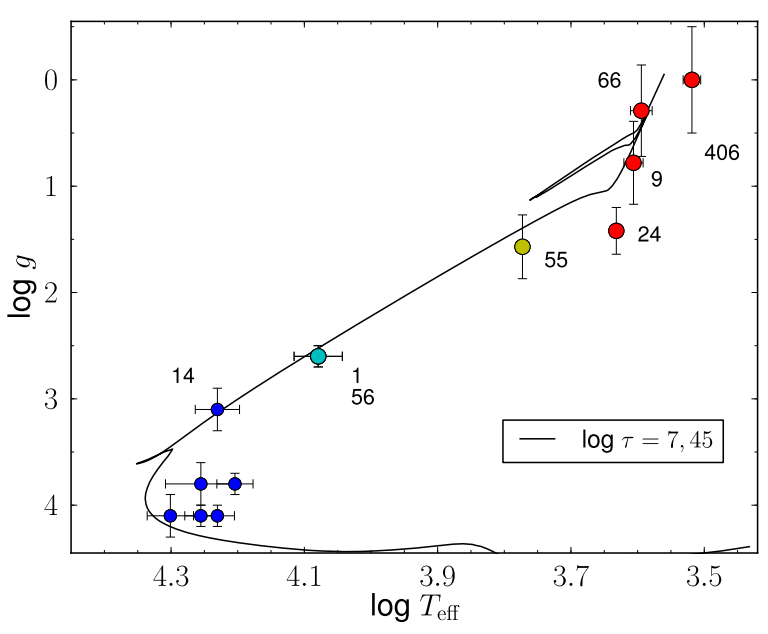

Fig. 11. Kiel diagram for likely members (with the exception of Be stars). Symbols and colours are as in Fig. 5. Stars 1 and 56 occupy the same position on the diagram.

iron lines, we were forced to modify our methodology. We followed the procedure described in García-Hernández et al. (2007), focusing on the region 6670-6730 $\AA$ where the TiO bands at 6681 and $6714 \AA$ are clearly present. These bands are very useful since their depth is very sensitive to temperature (García-Hernández et al. 2007). We used the same grid of MARCS synthentic spectra, but used TURBOSPECTRUM (Plez 2012) as a transfer code. Due to the strong degeneracy between metallicity and gravity, we fixed the former with the mean value derived from the FK supergiants. This is valid when the M star has a RV compatible with the cluster average. If not, we assumed a $\log g=0.0$, leaving the $T_{\text {eff }}$ as a free parameter.

The results (effective temperature, surface gravity, macroturbulent velocity, and iron abundance) are listed in Table 8. From the analysis of the three $\mathrm{K}$ and the $\mathrm{F}$ supergiant, we derive a subsolar metallicity for the cluster. The weighted arithmetic mean (using the variances as weights) is $[\mathrm{Fe} / \mathrm{H}]=-0.29 \pm 0.22$. The uncertainty, which is quite conservative, is expressed in terms of the standard deviation around this mean. We find a relatively high dispersion between our values for metallicity. Given that 
Table 9. Chemical abundances, relative to solar abundances by Grevesse et al. (2007), measured first on the cool stars and then only with stars 9 and 66, since star 24 might be polluted by its blue companion.

\begin{tabular}{lrrrrcc}
\hline \hline Star & \multicolumn{1}{c}{$[\mathrm{O} / \mathrm{H}]$} & \multicolumn{1}{c}{$[\mathrm{Na} / \mathrm{H}]$} & \multicolumn{1}{c}{$[\mathrm{Mg} / \mathrm{H}]$} & \multicolumn{1}{c}{$[\mathrm{Si} / \mathrm{H}]$} & {$[\mathrm{Ca} / \mathrm{H}]$} & \multicolumn{1}{c}{$[\mathrm{Ti} / \mathrm{H}]$} \\
\hline 9 & $-0.24 \pm 0.26$ & $-0.04 \pm 0.35$ & $-0.01 \pm 0.15$ & $0.11 \pm 0.32$ & $0.07 \pm 0.26$ & $0.21 \pm 0.32$ \\
24 & $0.26 \pm 0.17$ & $0.02 \pm 0.11$ & $0.02 \pm 0.11$ & $0.15 \pm 0.23$ & $0.38 \pm 0.20$ & $0.27 \pm 0.16$ \\
66 & $-0.48 \pm 0.27$ & $-0.07 \pm 0.45$ & $-0.12 \pm 0.14$ & $-0.06 \pm 0.36$ & $0.24 \pm 0.39$ & $0.07 \pm 0.45$ \\
Mean & $-0.02 \pm 0.44$ & $0.01 \pm 0.31$ & $-0.03 \pm 0.15$ & $0.09 \pm 0.32$ & $0.26 \pm 0.32$ & $0.24 \pm 0.33$ \\
Mean (no 24) & $-0.36 \pm 0.31$ & $-0.05 \pm 0.40$ & $-0.07 \pm 0.16$ & $0.03 \pm 0.36$ & $0.12 \pm 0.35$ & $0.16 \pm 0.40$ \\
Star & \multicolumn{1}{c}{$[\mathrm{Ni} / \mathrm{H}]$} & \multicolumn{1}{c}{$[\mathrm{Rb} / \mathrm{H}]$} & \multicolumn{1}{c}{$[\mathrm{Y} / \mathrm{H}]$} & \multicolumn{1}{c}{$[\mathrm{Ba} / \mathrm{H}]$} & $E W(\mathrm{Li})$ & $\mathrm{A}(\mathrm{Li})$ \\
\hline 9 & $0.00 \pm 0.34$ & -0.31 & $-0.28 \pm 0.30$ & $0.48 \pm 0.16$ & 164.2 & $<0.89$ \\
24 & $0.25 \pm 0.20$ & 0.05 & $0.09 \pm 0.40$ & $0.58 \pm 0.11$ & 99.0 & $<0.93$ \\
66 & $-0.15 \pm 0.37$ & -0.35 & $-0.33 \pm 0.52$ & $0.35 \pm 0.19$ & 122.9 & $<0.52$ \\
Mean & $0.13 \pm 0.36$ & $-0.20 \pm 0.22$ & $-0.18 \pm 0.47$ & $0.51 \pm 0.19$ & - & - \\
Mean (no 24) & $-0.07 \pm 0.37$ & $-0.33 \pm 0.03$ & $-0.29 \pm 0.41$ & $0.43 \pm 0.20$ & - & - \\
\hline
\end{tabular}

the star with higher metallicity, S24, has a blue companion that may affect the calculation, the actual cluster metallicity could be even lower.

Finally, in Fig. 11 we plot the Kiel diagram (i.e. $\log g_{-}$ $\left.\log T_{\text {eff }}\right)$ for likely members for which we have stellar parameters (Tables 7 and 8). In this diagram, independent of the cluster distance, we also plot the best-fitting isochrones according to that seen in Fig. 8. The isochrone, with the expected exceptions of the Be stars (7 and 72), reproduces quite well the observed evolutionary location of stars. It should be noted that supergiant stars (blue, yellow, and red) all lie very close to the isochrone. Only S24 (composite spectrum) and S406 (see further discussion in Sect. 4.5) fall away from it.

The good agreement between spectral results (stellar parameters) and cluster parameters, such as the distance and age inferred photometrically, supports the reliability of our results.

\subsection{Chemical abundances}

We derived chemical abundances for the three K-supergiants; for the remaining stars (including the YSG) there are no spectral diagnostic features whose quality is high enough for their analysis.

We employed the same methodology as in the study of NGC 6067 (Alonso-Santiago et al. 2017). We used a method based on $E W$ s measured in a semi-automatic fashion using TAME (Kang \& Lee 2012) for $\mathrm{Na}, \mathrm{Mg}, \mathrm{Si}, \mathrm{Ca}, \mathrm{Ti}, \mathrm{Ni}, \mathrm{Y}$, and $\mathrm{Ba}$. We also measured $E W \mathrm{~s}$ by hand for two special and delicate cases, namely oxygen and lithium, using the IRAF SPLOT task. For lithium, we employed a classical analysis using the $6707.8 \AA$ line, taking into account the nearby Fe I line at $6707.4 \AA$. We measured the $E W$ by hand (in $\mathrm{m} \AA$ ) using the IRAF SPLOT task. We use the standard notation, where $A(\mathrm{Li})=\log [n(\mathrm{Li}) / n(\mathrm{H})]+12$. In the case of oxygen, we used the [O I] $6300 \AA$ line. This oxygen line is blended with a Ni I feature; we corrected the $E W \mathrm{~s}$ accordingly by using the methodology and line atomic parameters described in Bertran de Lis et al. (2015). Finally, we also derived rubidium abundances using stellar synthesis for the $7800 \AA \mathrm{Rb}$ I line, following the methodology in D'Orazi et al. (2013).

Table 9 summarises the abundances found for each star together with their errors. We estimate the cluster average by using a weighted arithmetic mean (employing the variances as weights). We computed conservative errors since the associated uncertainty is the combination in quadrature of the individual typical error and the star-to-star dispersion. This average has been computed both with and without star 24 since it has a blue companion. Although we do not see any trace of the companion in the spectral range used for the analysis, we cannot discard the possibility that it is affecting the results given that the values found are quite different from those of stars 9 and 66, which have very similar spectra.

\section{Discussion}

\subsection{Cluster parameters}

Unlike previous works, it should be noted that our photometric analysis has been strengthened by including spectroscopy. In addition, we have employed the largest number of likely members to date. In Table 10, we summarise our values for cluster parameters and compare them with those in the literature. The amount of reddening is compatible within the errors with the values calculated by previous authors, with the exception of Paunzen et al. (2005), who used the $\Delta a$ photometric system instead of Johnson's. Our determination of a low metallicity for the cluster implies that it is slightly older and is located somewhat closer than in previous works which assumed solar composition. With respect to the first studies, based on photographic and photoelectric data, we observe a larger cluster size. Our result is consistent with the membership of S66, a radial velocity member, that is located at around 2.2 from the cluster centre. Moreover, we provide a new value for the cluster coordinates $\left(10^{\mathrm{h}} 00^{\mathrm{m}} 41^{\mathrm{s}},-54^{\circ} 47^{\prime} 21^{\prime \prime}\right)$ very close to the nominal ones, with a small offset (this work - nominal) of $\Delta \alpha \approx 2^{\prime \prime}$ and $\Delta \delta \approx 3^{\prime \prime}$.

NGC 3105 , located at $R_{\mathrm{GC}} \sim 10 \mathrm{kpc}$ and presenting a $[\mathrm{Fe} / \mathrm{H}] \sim-0.3$, is a distant and metal-poor cluster. It is located at a position compatible with that expected from its observed radial velocity, computed by us for the first time. According to the flat rotation curve for the Galaxy (Reid et al. 2009), in the line-of-sight direction to the cluster, the expected distance corresponding to the observed $\mathrm{RV}$ is $7.0 \mathrm{kpc}$, compatible within the errors with the value found in this work, $d=7.2 \pm 0.7 \mathrm{kpc}$. In addition, we estimated, also for the first time, the initial mass for this cluster obtaining a value around $4000 M_{\odot}$, which makes NGC 3105 a moderately massive cluster.

Given its location in the Galaxy $\left(l \sim 280^{\circ}, d \sim 7 \mathrm{kpc}\right)$ NGC 3105 is young enough to serve as a tracer of the Sagittarius-Carina arm. However, in this direction, and at 
Table 10. Summary of cluster parameters for NGC 3105 derived in this work compared to studies found in the literature.

\begin{tabular}{lccccc}
\hline \hline Reference & Phot. & $E(B-V)$ & $d(\mathrm{kpc})$ & Age $(\mathrm{Ma})$ & $r_{\mathrm{cl}}(\mathrm{pc})$ \\
\hline Moffat \& Fitzgerald (1974) & $\mathrm{Pg}+\mathrm{Pe}$ & $1.09 \pm 0.03$ & $8.0 \pm 1.5$ & - & 2.6 \\
Fitzgerald et al. (1977) & Pe & $1.08 \pm 0.03$ & $5.5 \pm 0.8$ & - & 1.8 \\
Sagar et al. (2001) & CCD & 1.06 & $9.5 \pm 1.5$ & $25 \pm 10$ & - \\
Paunzen et al. (2005) & CCD & $0.95 \pm 0.02$ & $8.5 \pm 1.0$ & $20 \pm 5$ & - \\
This work & CCD & $1.03 \pm 0.03$ & $7.2 \pm 0.7$ & $28 \pm 6$ & $5.8 \pm 1.7$ \\
\hline
\end{tabular}

similar distances, there are no clusters with known metallicity with which we can compare our results.

In NGC 3105 we find RSGs with masses around $9.5 M_{\odot}$. According to most modern evolutionary tracks, stars of this mass at this metallicity, will end their lives in a core-collapse supernova explosion, but are just above the minimum mass required for this to happen (see Doherty et al. 2017, and references therein). Star 33, for which unfortunately we lack a high-resolution spectrum, is definitely an M-type supergiant, while stars 9, 24, and 66 are $\mathrm{K}$ types. In a sample of clusters with ages between 50 and $100 \mathrm{Ma}$, all the evolved red stars (which will become AGB stars according to models) are K types (Negueruela et al. 2017; see also Negueruela \& Marco 2012). In clusters somewhat younger than NGC 3105, such as NGC 3766 or NGC 663, there are only M-type supergiants. This is in agreement with the results of Mermilliod (1981) who found that the average spectral type of red (super)giants becomes later with younger ages. The comparison, however, is not straightforward because the typical spectral types of RSGs depend on metallicity (Levesque et al. 2006; Dorda et al. 2016). In fact, the spectral distribution of the RSGs in NGC 3105 is similar to that of moderate- to low-luminosity RSGs in the LMC, which presents a similar average metallicity (Dorda et al. 2016). It seems then that the stars in NGC 3105 are among the lowest-mass stars that will give rise to a core-collapse supernova.

\subsection{Blue-to-red supergiant ratio}

The blue supergiants to red supergiants $(B / R)$ ratio is one of the most important problems in high-mass stellar evolution. Current models fail when trying to reproduce the observed ratios at different metallicities (Langer \& Maeder 1995; Dohm-Palmer \& Skillman 2002). This ratio is an excellent test for stellar models because it is very sensitive to the input physics: mass loss, convection, and mixing process (Langer \& Maeder 1995) or rotation (Maeder \& Meynet 2001). The value of $B / R$ seems to be directly proportional to metallicity (Langer \& Maeder 1995; Eggenberger et al. 2002), with consequences such as the nature of the supernova progenitors in different environments (Langer 1991), the study of the luminous star population in other galaxies (Langer \& Maeder 1995; Origlia et al. 1999), or the variation in the period of variable stars (Maeder \& Meynet 2001).

According to Eggenberger et al. (2002), who studied this ratio in young clusters at different metallicities in the Milky Way (MW) and the Magellanic Clouds (SMC and LMC), $B / R$ as a function of metallicity is given by

$$
\frac{B / R}{(B / R)_{\odot}}=0.05 \cdot \mathrm{e}^{3 \cdot \frac{z}{z_{\odot}}} \text {, }
$$

where $B$ is the number of BSGs (including $\mathrm{O}, \mathrm{B}$, and A supergiants), $R$ is the number of RSGs (K and $\mathrm{M}),(B / R)_{\odot} \cong 3$, and $Z_{\odot}=0.02$. In our case, for NGC 3105 (not included in the work of Eggenberger et al. 2002), $Z \cong 0.0103$ (referring to the solar value just mentioned, $\left.Z_{\odot}=0.02\right)$ and so we would expect an 'empirical' ratio $(B / R)_{\mathrm{emp}} \approx 0.70$. Since the cluster hosts two A supergiants and five RSGs $(3 \mathrm{~K}+2 \mathrm{M})$, the observed ratio is $(B / R)_{\mathrm{obs}}=0.4$. This value is slightly higher than half of the expected empirical value, though caution should be used because of the low statistical significance of our sample.

Another interesting observable used to constrain theoretical models is the yellow-to-red supergiant $(\mathrm{Y} / \mathrm{R})$ ratio. This ratio is very sensitive to the extension of the blue loop (where YSGs spend most of the time during this phase, according to Anderson 2014). The blue loop depends strongly on the metallicity and the input physics (Matraka et al. 1982; Ekström et al. 2012; Walmswell et al. 2015). NGC 3105 contains one YSG and five RSGs, i.e. $(\mathrm{Y} / \mathrm{R})=0.2$. This ratio is typically found in clusters within a similar age range such as NGC 457, NGC 654, NGC 6649, NGC 6664, or Berkeley 55 (Sowell 1987; Negueruela \& Marco 2012, and references therein). However, this value is lower than those predicted by stellar evolution models.

\subsection{Be stars}

Classical Be stars are non-supergiant B-type stars that show or have shown Balmer lines in emission at any time (Porter \& Rivinius 2003). This phenomenon is related to fast rotation, which generates circumstellar discs around the star, responsible for the emission observed. Be stars are usually located around the MSTO of young open clusters (McSwain \& Gies 2005). They are observed in clusters covering the whole Btype range. Mermilliod (1982) suggested that the Be fraction, i.e. $\mathrm{Be} /(\mathrm{B}+\mathrm{Be})$, decreases with increasing age. $\mathrm{He}$ found a $\mathrm{Be}$ star distribution with two peaks: one at B1-B2 and the other at B7-B8. According to theoretical considerations concerning rotational velocity distribution (Granada et al. 2016), the largest fraction of Be stars should occur in populations ranging from $\approx 16$ to $40 \mathrm{Ma}$. Granada et al. (2016) assume that the Be phenomenon is observed above $\Omega / \Omega_{\text {crit }} \geq 0.8$. In addition, the Be fraction is around three times higher in early $B$ stars. These results seem to agree with observations: NGC 663 (Pigulski et al. 2001) or NGC 7419 (Marco \& Negueruela 2013), with ages in the 15-25 Ma range, are among the clusters with the highest Be fractions (up to $\approx 40 \%$ ). On the other hand, McSwain \& Gies (2005) performed a detailed analysis of a sample containing 55 open clusters, finding a broad maximum for the Be fraction for ages between 25 and $100 \mathrm{Ma}$.

In the field of NGC 3105 we find 11 Be stars, of which we consider 9 to be likely members. Most of them are located in the upper main sequence, close to the MSTO. We find seven Be stars between the top of the MS and spectral type b3. This number implies a Be fraction of $35 \%$ for stars brighter than $V=16.3$, corresponding to spectral type B3 V, or $25 \%$ when considering likely members with spectral types down to b3. Tarasov \& Malchenko (2012) studied the relative content of Be stars in 42 young clusters aged up to $35 \mathrm{Ma}$, taking into 
account objects in the B0-B3 range alone. The maximum frequency of Be stars occurs in clusters with ages from 13 to $25 \mathrm{Ma}$, reaching a $\mathrm{Be}$ fraction of around $25 \%$. For the age range of NGC 3105 (in their Fig. 2, the 25-30 Ma bin) they found a fraction of $15 \%$, almost half that found by us in NGC 3105. Hence, NGC 3105 (not included in their work) is among the clusters with the highest Be fractions together with well-known clusters such as $\mathrm{h} / \chi$ Persei (Malchenko \& Tarasov 2008) or NGC 7419 (Marco \& Negueruela 2013). However, these clusters are younger than NGC 3105, with ages around 12-14 Ma.

The shape of the cluster can be taken as a clue to the initial angular momentum with which the cluster originated. According to Keller et al. (1999) the ellipsoidal shape of the SMC massive cluster NGC 330 would be the consequence of having been formed from material with high angular momentum. This would explain the high Be fraction observed in that cluster, related to a rapid rotation rate. This same argument might be used as an explanation for the high Be frequency in NGC 3105, a cluster with a high eccentricity $(\epsilon=0.51$, see Sect. 3.3). However, we are cautious since this ellipsoidal shape could also be the result of a previous merger of two subclusters.

Finally, the Be phenomenon is also related to metallicity. The Be fraction increases with decreasing metallicity (Maeder \& Meynet 2000; Martayan et al. 2007). According to Maeder \& Meynet (2000), in low-metallicity environments, mass loss via a radiative wind is reduced and is thus not very effective at breaking down fast rotation. Martayan et al. (2007) studied the ratios $\Omega / \Omega_{\text {crit }}$ for Be stars in several galaxies with different metallicities. They found for the MW (assuming $Z=$ $0.020)$, LMC $(Z=0.004)$, and SMC $(Z=0.002) \Omega / \Omega_{\text {crit }}$ values of $0.80,0.85$, and 0.95 , respectively. In these galaxies they also studied the B and Be populations and noted for field Be stars fractions of $15 \%, 17.5 \%$, and $26 \%$. As seen, the frequency of $\mathrm{Be}$ stars is inversely related to the average metallicity. NGC 3105 has a particularly low metallicity for a Milky Way young cluster, and seems to fit this trend.

\subsection{Low metallicity}

In the current work, for the first time, chemical abundances for NGC 3105 are provided. In the absence of previous work with which to compare our results, we place the cluster on the Galactic gradient. As a reference we use the work by Genovali et al. (2013, 2014). They used Cepheids to estimate the radial distribution of metallicity (in terms of $[\mathrm{Fe} / \mathrm{H}]$ ), as this is a young population $(\tau \approx 20-400 \mathrm{Ma})$ tracing present-day abundances, and thus provides a good comparison for young clusters. In Fig. 12, we show the Galactic gradient by Genovali et al. (2013, 2014) with the position of NGC 3105 overplotted, and a sample of open clusters taken from Netopil et al. (2016) for further comparison. We selected young clusters $(\tau<500 \mathrm{Ma})$ whose metallicity is obtained from high-quality spectra (Heiter et al. 2014) as the average of at least three stars. It is obvious that NGC 3105 is a metal-poor cluster.

We also compare our abundances with the Galactic trends for the thin disc (Fig. 13). We plot abundance ratios of $[\mathrm{X} / \mathrm{Fe}]$ versus $[\mathrm{Fe} / \mathrm{H}]$ obtained by Adibekyan et al. (2012) for $\mathrm{Na}, \mathrm{Mg}$, $\mathrm{Si}, \mathrm{Ca}, \mathrm{Ti}$, and Ni and by Delgado Mena et al. (2017) for Y and $\mathrm{Ba}$ in the workframe of the HARPS GTO planet search program. The chemical composition of NGC 3105 is compatible, within the errors, with the Galactic trends observed in the thin disc of the Galaxy. No significant amounts of either Li or Rb have been found in the RSGs. We derived a roughly solar [Y/Fe] against a supersolar $[\mathrm{Ba} / \mathrm{Fe}]$, which is in good agreement with the

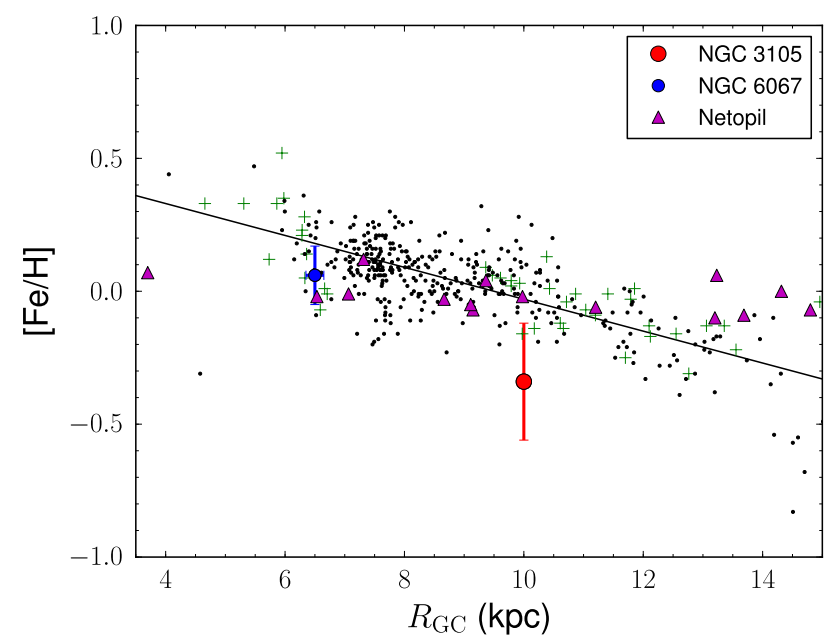

Fig. 12. Iron abundance gradient in the Milky Way found by Genovali et al. $(2013,2014)$. The black line is the Galactic gradient $\left(-0.06 \mathrm{dex} \mathrm{kpc}^{-} 1\right)$, green crosses are Cepheids studied in those papers, whereas black dots show data for other Cepheids from the literature used by these authors. Magenta triangles represent the young open clusters $(<500 \mathrm{Ma})$ in the sample compiled by Netopil et al. (2016). Finally, the red circle is NGC 3105 and the blue one is NGC 6067. Both young clusters were analysed by our group with the same techniques. NGC 3105 falls well below the metallicity typically observed at its Galactocentric distance. All the values shown in this plot are rescaled to Genovali et al. (2014), i.e. $R_{\odot}=7.95 \mathrm{kpc}$ and $\mathrm{A}(\mathrm{Fe})=7.50$.

dependence on age and Galactic location found by Mishenina et al. (2013) by comparing the abundances of $\mathrm{Y}$ and $\mathrm{Ba}$ in different open clusters. Remarkably, we find a strong overabundance of barium $([\mathrm{Ba} / \mathrm{Fe}]=+0.8)$. Several young open clusters have been observed to have $\mathrm{Ba}$ abundances higher than those predicted by standard theoretical models. To explain this enrichment of Ba, D'Orazi et al. (2009) suggested an enhanced 's-process'. Our result supports this idea (see their Fig. 2).

\subsection{The nature of star 406}

Star 406 has been found to be a luminous late-M star that we have classified as M6 Ib, even though luminosity criteria are not well defined at these spectral types (cf. Negueruela et al. 2012). The star has a radial velocity fully compatible with cluster membership, and has thus been included as a cluster member. However, we must note that we know of no other star with a similar spectral type that is a member of an open cluster of this age. Two massive young clusters, Stephenson 2 (16-20 Ma) and NGC 7419 ( $\approx 14 \mathrm{Ma})$, contain stars with spectral types M6-7 that are clearly supergiants. These objects, however, are much brighter than other RSGs in those clusters, and show evidence for very heavy mass loss. Star 406 is fainter in $K_{\mathrm{S}}$ than the other Mtype supergiant, S33. Using the $\left(K_{\mathrm{S}}-W 3\right)$ colour as an indication of mass loss, it does not seem to have a significantly higher mass loss than the other RSGs $(0.38$ mag against 0.30 mag for S33 or 0.33 for S66). However, interpretation of the data is difficult, as S33 is almost one magnitude brighter than the next brightest RSG, S66. The possibility that S406 is an unrelated luminous red giant with exactly the same radial velocity falling within the tidal radius of the cluster is slim. Given the masses of the evolved stars, close to the boundary between intermediate- and highmass stars, we can speculate that S406 might be a super-AGB star (i.e. a star of $\gtrsim 8 M_{\odot}$ that can ignite carbon off-centre but will still experience a third dredge-up; Poelarends et al. 2008). 

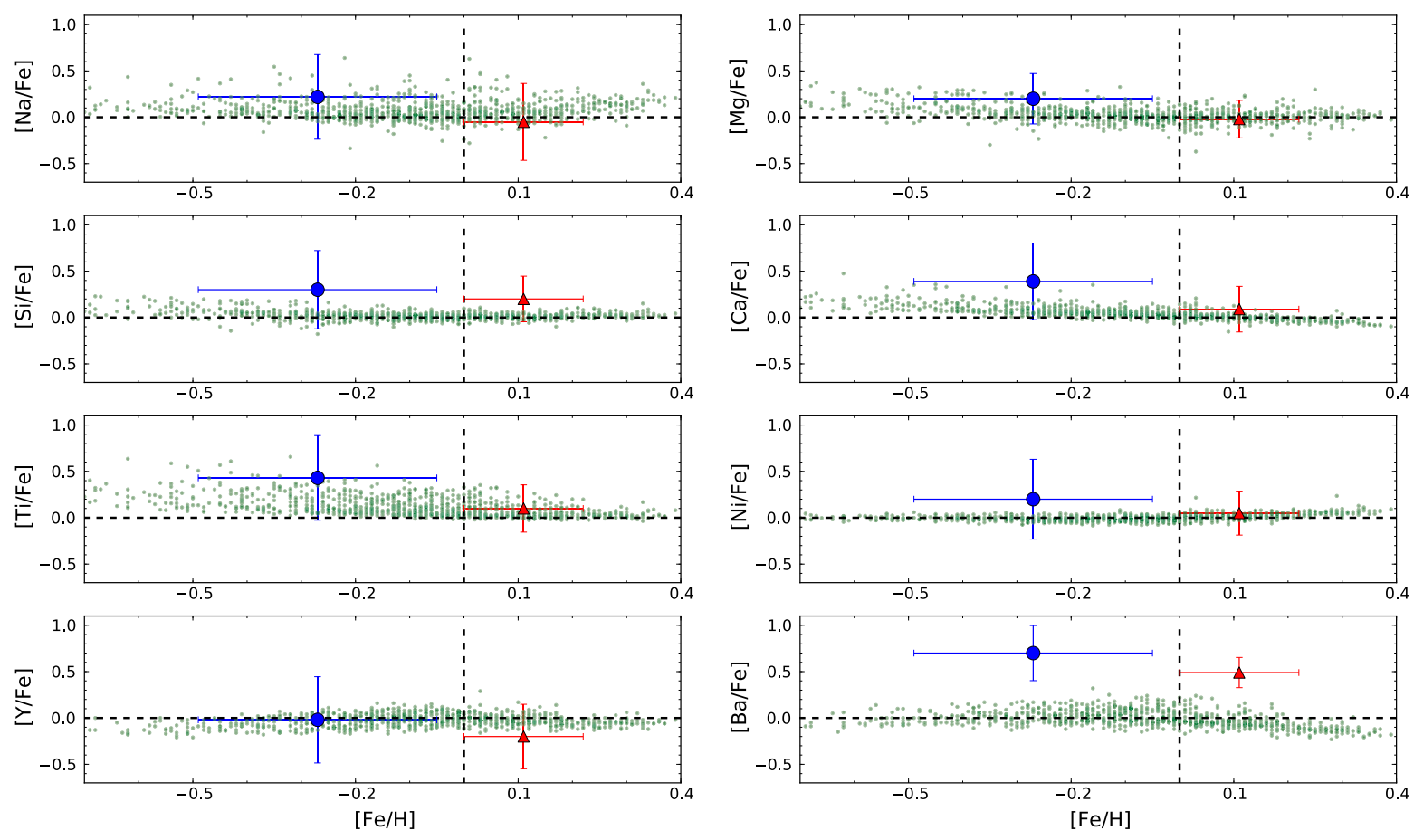

Fig. 13. Abundance ratios $[\mathrm{X} / \mathrm{Fe}]$ versus $[\mathrm{Fe} / \mathrm{H}]$. The green dots represent the galactic trends for the thin disc (Adibekyan et al. 2012; Delgado Mena et al. 2017). NGC 6067 is the red triangle, whereas NGC 3105 (without S24) is the blue circle. Clusters are represented by their mean values. The dashed lines indicate the solar value.

Against this interpretation, we note that S406 does not show any evidence of the presence of $\mathrm{Li}$ or $\mathrm{Rb}$, which are seen in many massive AGB stars at LMC typical metallicity and similar temperatures (García-Hernández et al. 2009).

\section{Conclusions}

We have performed the most complete analysis to date of NGC 3105, a cluster poorly studied until now. Our photometry is deeper than previous studies and no significant offsets with them are noted. The cluster parameters obtained in this work, $E(B-V)=1.03, d=7.2 \mathrm{kpc}, \tau=27 \mathrm{Ma}$, are compatible within the errors with those found in the literature.

We estimate a cluster size, $r_{\mathrm{cl}} \approx 2.7 \mathrm{arcmin}$, doubling or even tripling previous estimations. This size is consistent with the location of the most distant members confirmed via radial velocity. We identify 126 likely blue members, from which we derive an initial mass slightly above $4000 M_{\odot}$. Therefore, NGC 3105 is a moderately massive cluster, as expected from a population of eight supergiant stars, spanning the blue, yellow, and red phase, a rare occurrence.

NGC 3105 is comparable to NGC 7419 as a template for studying obscured RSGCs and for constraining evolutionary models of intermediate- and high-mass stars. Evolved stars in NGC 3105 are set in the lower limit of massive stars. Its RSGs, with masses around $9.5 M_{\odot}$, are just above the minimum mass to explode as a supernova. As in NGC 7419, we find a cluster with less than $10000 M_{\odot}$ (in this case, significantly less), that still contains five RSGs (although one is not a certain member).

As in other clusters, we find that the locations of the A-type supergiants in both the colour-magnitude and Kiel diagrams indicate that they are just leaving the MS and are not blue-loop objects. Current Geneva models (Georgy et al. 2013) suggest that most A-type supergiants are He-core burning objects in the blue loop, but there is little observational evidence for this. Even the F-type supergiant seems to be in the Hertzsprung gap, where no stars are expected, rather than in the blue loop.

Based on good S/N and high-resolution spectra, we infer a cluster $v_{\text {rad }}=+46.9 \mathrm{~km} \mathrm{~s}^{-1}$ and derive a subsolar metallicity, $[\mathrm{Fe} / \mathrm{H}]=-0.29$. At the cluster Galactocentric distance $\left(R_{\mathrm{GC}}=\right.$ $10.0 \mathrm{kpc}$ ) this value is rather below the expected value according to the Galactic gradient. This low metallicity and its elliptical geometry would explain the high fraction $(\approx 25 \%)$ of Be stars found from the stars observed with the earliest spectral types down to spectral type b3.

For the first time, we have calculated stellar parameters for hot and cool stars within the cluster. As shown in the Kiel diagram, the parameters obtained for the two sets of stars, using different codes and techniques, agree well with each other (as they trace a single theoretical isochrone) and also with the results derived from photometry (as it is the isochrone corresponding to the photometric age). For the first time we have also determined the chemical abundances of the $\mathrm{Li}, \mathrm{O}, \mathrm{Na}, \alpha$-elements $(\mathrm{Mg}, \mathrm{Si}, \mathrm{Ca}$, and $\mathrm{Ti}$ ); the Fe-group ( $\mathrm{Ni})$; and the $s$-elements ( $\mathrm{Rb}, \mathrm{Y}$, and $\mathrm{Ba})$. The overabundance of $\mathrm{Ba}$ found supports the $s$-enhanced process.

The determination of the metallicity of a young cluster is fundamental for its correct characterisation. The use of solar metallicity isochrones leads to an incorrect estimation of the age and the mass of the RSGs. Despite its unusually low metallicity, NGC 3105 is an excellent laboratory for improving theoretical models. The derivation of stellar parameters for its blue supergiants and its brightest RSG, star 33, would provide an excellent complement to this work and would improve the value of NGC 3105 as a template for more obscured or unresolved clusters.

Acknowledgements. We thank the anonymous referee for the helpful suggestions which have helped to improve this paper. We also thank Aníbal García Hernández for instruction in the use of TuRBospectrum. This research is partially supported by the Spanish Government Ministerio de Economía y Competitividad under grants BES 2013-065384 and AYA2015-68012-C2-2$\mathrm{P}$ (MINECO/FEDER). H.M.T. would like to thank the Spanish Government 
(Ministerio de Economía y Competitividad) for the support under grant FJCI2014-23001. The research leading to these results has received funding from the European Community's Seventh Framework Programme (FP7/2013-2016) under grant agreement number 312430 (OPTICON); proposal number 2015A/008 This paper uses observations made at the South African Astronomical Observatory (SAAO). This research has made use of the Simbad database, operated at CDS, Strasbourg (France). This publication has also made use of data products from the Two Micron All Sky Survey, which is a joint project of the University of Massachusetts and the Infrared Processing and Analysis Center/California Institute of Technology, funded by the National Aeronautics and Space Administration and the National Science Foundation.

\section{References}

Adibekyan, V. Z., Sousa, S. G., Santos, N. C., et al. 2012, A\&A, 545, A32

Allen, C. W. 1973, Astrophysical Quantities (London: University of London, Athlone Press)

Aller, L. H., Appenzeller, I., Baschek, B., et al., eds. 1982, Landolt-Börnstein: Numerical Data and Functional Relationships in Science and Technology New Series Vol. 2, 54

Alonso-Santiago, J., Negueruela, I., Marco, A., et al. 2017, MNRAS, 469, 1330 Anderson, R. I. 2014, A\&A, 566, L10

Barklem, P. S., Piskunov, N., \& O’Mara, B. J. 2000, A\&AS, 142, 467

Barnsley, R. M., \& Steele, I. A. 2013, A\&A, 556, A81

Bertran de Lis, S., Delgado Mena, E., Adibekyan, V. Z., Santos, N. C., \& Sousa, S. G. 2015, A\&A, 576, A89

Blanco-Cuaresma, S., Soubiran, C., Heiter, U., \& Jofré, P. 2014, A\&A, 569, A111

Bressan, A., Marigo, P., Girardi, L., et al. 2012, MNRAS, 427, 127

Buzzoni, B., Delabre, B., Dekker, H., et al. 1984, The Messenger, 38, 9

Carquillat, M. J., Jaschek, C., Jaschek, M., \& Ginestet, N. 1997, A\&AS, 123

Castro, N., Urbaneja, M. A., Herrero, A., et al. 2012, A\&A, 542, A79

Clark, J. S., Negueruela, I., Crowther, P. A., \& Goodwin, S. P. 2005, A\&A, 434 949

Clark, J. S., Davies, B., Najarro, F., et al. 2009a, A\&A, 504, 429

Clark, J. S., Negueruela, I., Davies, B., et al. 2009b, A\&A, 498, 109

Crowther, P. A., Schnurr, O., Hirschi, R., et al. 2010, MNRAS, 408, 731

Davies, B., Figer, D. F., Kudritzki, R.-P., et al. 2007, ApJ, 671, 781

Delgado Mena, E., Tsantaki, M., Adibekyan, V. Z., et al. 2017, A\&A, 606, A94

Doherty, C. L., Gil-Pons, P., Siess, L., \& Lattanzio, J. C. 2017, PASA, 34, e056

Dohm-Palmer, R. C., \& Skillman, E. D. 2002, AJ, 123, 1433

D’Orazi, V., Magrini, L., Randich, S., et al. 2009, ApJ, 693, L31

D'Orazi, V., Lugaro, M., Campbell, S. W., et al. 2013, ApJ, 776, 59

Dorda, R., Negueruela, I., González-Fernández, C., \& Tabernero, H. M. 2016 A\&A, 592, A16

Draper, P. W., Taylor, M., \& Allan, A. 2011, Starlink User Note, 139

Dunstall, P. R., Dufton, P. L., Sana, H., et al. 2015, A\&A, 580, A93

Dutra-Ferreira, L., Pasquini, L., Smiljanic, R., Porto de Mello, G. F., \& Steffen, M. 2016, A\&A, 585, A75

Eggenberger, P., Meynet, G., \& Maeder, A. 2002, A\&A, 386, 576

Ekström, S., Georgy, C., Eggenberger, P., et al. 2012, A\&A, 537, A146

Fernie, J. D. 1963, AJ, 68, 780

Fitzgerald, M. P. 1970, A\&A, 4, 234

Fitzgerald, M. P., Moffat, A. F. J., \& Jackson, P. D. 1977, A\&A, 59, 141

Foreman-Mackey, D., Hogg, D. W., Lang, D., \& Goodman, J. 2013, PASP, 125 306

García-Hernández, D. A., García-Lario, P., Plez, B., et al. 2007, A\&A, 462, 711

García-Hernández, D. A., Manchado, A., Lambert, D. L., et al. 2009, ApJ, 705 L31

Genovali, K., Lemasle, B., Bono, G., et al. 2013, A\&A, 554, A132

Genovali, K., Lemasle, B., Bono, G., et al. 2014, A\&A, 566, A37

Georgy, C., Ekström, S., Granada, A., et al. 2013, A\&A, 553, A24

González-Fernández, C., \& Negueruela, I. 2012, A\&A, 539, A100

Granada, A., Georgy, C., Haemmerlé, L., Ekström, S., \& Meynet, G. 2016, ASP Conf. Ser., 506, 33

Gray, R. O., \& Corbally, C. J. 1994, AJ, 107, 742

Gray, R. O., \& Corbally, J. C. 2009, Stellar Spectral Classification (Princeton: Princeton University Press)

Grevesse, N., Asplund, M., \& Sauval, A. J. 2007, Space Sci. Rev., 130, 105

Gustafsson, B., Edvardsson, B., Eriksson, K., et al. 2008, A\&A, 486, 951

Heiter, U., \& Eriksson, K. 2006, A\&A, 452, 1039

Heiter, U., Soubiran, C., Netopil, M., \& Paunzen, E. 2014, A\&A, 561, A93

Humphreys, R. M., \& McElroy, D. B. 1984, ApJ, 284, 565

Jaschek, C., \& Gómez, A. E. 1970, PASP, 82, 809

Jaschek, C., \& Jaschek, M. 1987, The Classification of Stars (Cambridge: University Press)
Johnson, H. L. 1958, Lowell Observatory Bulletin, 4, 37

Johnson, H. L., \& Morgan, W. W. 1953, ApJ, 117, 313

Kang, W., \& Lee, S.-G. 2012, MNRAS, 425, 3162

Kaufer, A., Stahl, O., Tubbesing, S., et al. 1999, The Messenger, 95, 8

Keller, S. C., Wood, P. R., \& Bessell, M. S. 1999, A\&AS, 134, 489

Kouwenhoven, M. B. N., Brown, A. G. A., Zinnecker, H., Kaper, L., \& Portegies Zwart, S. F. 2005, A\&A, 430, 137

Kroupa, P. 2001, MNRAS, 322, 231

Kupka, F. G., Ryabchikova, T. A., Piskunov, N. E., Stempels, H. C., \& Weiss,

W. W. 2000, Balt. Astron., 9, 590

Landolt, A. U. 1992, AJ, 104, 340

Langer, N. 1991, A\&A, 252, 669

Langer, N., \& Maeder, A. 1995, A\&A, 295, 685

Lefever, K. 2007, PhD Thesis, Institute of Astronomy, Katholieke Universiteit Leuven, Belgium

Levesque, E. M., Massey, P., Olsen, K. A. G., et al. 2005, ApJ, 628, 973

Levesque, E. M., Massey, P., Olsen, K. A. G., et al. 2006, ApJ, 645, 1102

Lynga, G., \& Wramdemark, S. 1973, A\&AS, 12, 365

Maeder, A., \& Meynet, G. 2000, A\&A, 361, 159

Maeder, A., \& Meynet, G. 2001, A\&A, 373, 555

Malchenko, S. L., \& Tarasov, A. E. 2008, Astrophysics, 51, 250

Marco, A., \& Negueruela, I. 2013, A\&A, 552, A92

Marco, A., Negueruela, I., \& McBride, V. 2013, in Massive Stars: From Alpha to Omega, 166

Marco, A., Negueruela, I., González-Fernández, C., et al. 2014, A\&A, 567, A73

Marrese, P. M., Boschi, F., \& Munari, U. 2003, A\&A, 406, 995

Martayan, C., Frémat, Y., Hubert, A.-M., et al. 2007, A\&A, 462, 683

Matraka, B., Wassermann, C., \& Weigert, A. 1982, A\&A, 107, 283

McSwain, M. V., \& Gies, D. R. 2005, ApJS, 161, 118

Mermilliod, J. C. 1981, A\&A, 97, 235

Mermilliod, J-C. 1982, A\&A, 109, 37

Meynet, G., Georgy, C., Hirschi, R., et al. 2011, Bull. Soc. Roy. Sci. Liège, 80, 266

Mink, D. J. 2002, ASP Conf. Ser., 281, 169

Mishenina, T., Korotin, S., Carraro, G., Kovtyukh, V. V., \& Yegorova, I. A. 2013, MNRAS, 433, 1436

Moffat, A. F. J., \& Fitzgerald, M. P. 1974, A\&AS, 16, 25

Monguió, M., Figueras, F., \& Grosbø1, P. 2013, A\&A, 549, A78

Monguió, M., Negueruela, I., Marco, A., et al. 2017, MNRAS, 466, 3636

Negueruela, I., \& Marco, A. 2012, AJ, 143, 46

Negueruela, I., González-Fernández, C., Marco, A., \& Clark, J. S. 2011, A\&A, 528, A59

Negueruela, I., Marco, A., González-Fernández, C., et al. 2012, A\&A, 547, A15

Negueruela, I., Alonso-Santiago, J., Tabernero, H. M., et al. 2017, Mem. Soc. Astron. It., 88, 368

Netopil, M., Paunzen, E., \& Stütz, C. 2012, Astrophys. Space Sci. Proc., 29, 53

Netopil, M., Paunzen, E., Heiter, U., \& Soubiran, C. 2016, A\&A, 585, A150

Origlia, L., Goldader, J. D., Leitherer, C., Schaerer, D., \& Oliva, E. 1999, ApJ, 514, 96

Paunzen, E., Netopil, M., Iliev, I. K., et al. 2005, A\&A, 443, 157

Pigulski, A., Kopacki, G., \& Kołaczkowski, Z. 2001, A\&A, 376, 144

Piskunov, N. E., Kupka, F., Ryabchikova, T. A., Weiss, W. W., \& Jeffery, C. S. 1995, A\&AS, 112, 525

Plez, B. 2012, Astrophysics Source Code Library [record ascl:1205.004]

Poelarends, A. J. T., Herwig, F., Langer, N., \& Heger, A. 2008, ApJ, 675, 614

Porter, J. M., \& Rivinius, T. 2003, PASP, 115, 1153

Puls, J., Urbaneja, M. A., Venero, R., et al. 2005, A\&A, 435, 669

Reid, M. J., Menten, K. M., Zheng, X. W., et al. 2009, ApJ, 700, 137

Sagar, R., Munari, U., \& de Boer, K. S. 2001, MNRAS, 327, 23

Santolaya-Rey, A. E., Puls, J., \& Herrero, A. 1997, A\&A, 323, 488

Shortridge, K., Meyerdierks, H., Currie, M. J., et al. 2014, Astrophysics Source Code Library [record ascl:1411.022]

Simón-Díaz, S., \& Herrero, A. 2014, A\&A, 562, A135

Simón-Díaz, S., Castro, N., Herrero, A., et al. 2011, J. Phys. Conf. Ser., 328, 012021

Skrutskie, M. F., Cutri, R. M., Stiening, R., et al. 2006, AJ, 131, 1163

Slesnick, C. L., Hillenbrand, L. A., \& Massey, P. 2002, ApJ, 576, 880

Sowell, J. R. 1987, ApJS, 64, 241

Stetson, P. B. 1987, PASP, 99, 191

Straižys, V. 1992, Multicolor Stellar Photometry (Tucson: Pachart Pub. House)

Tabernero, H. M., Dorda, R., Negueruela, I., \& González-Fernández, C. 2018 MNRAS, 476, 3106

Tadross, A. L. 2005, Astron. Nachr., 326, 19

Tarasov, A. E., \& Malchenko, S. L. 2012, Astron. Lett., 38, 428

Walborn, N. R., \& Fitzpatrick, E. L. 1990, PASP, 102, 379

Walmswell, J. J., Tout, C. A., \& Eldridge, J. J. 2015, MNRAS, 447, 2951 


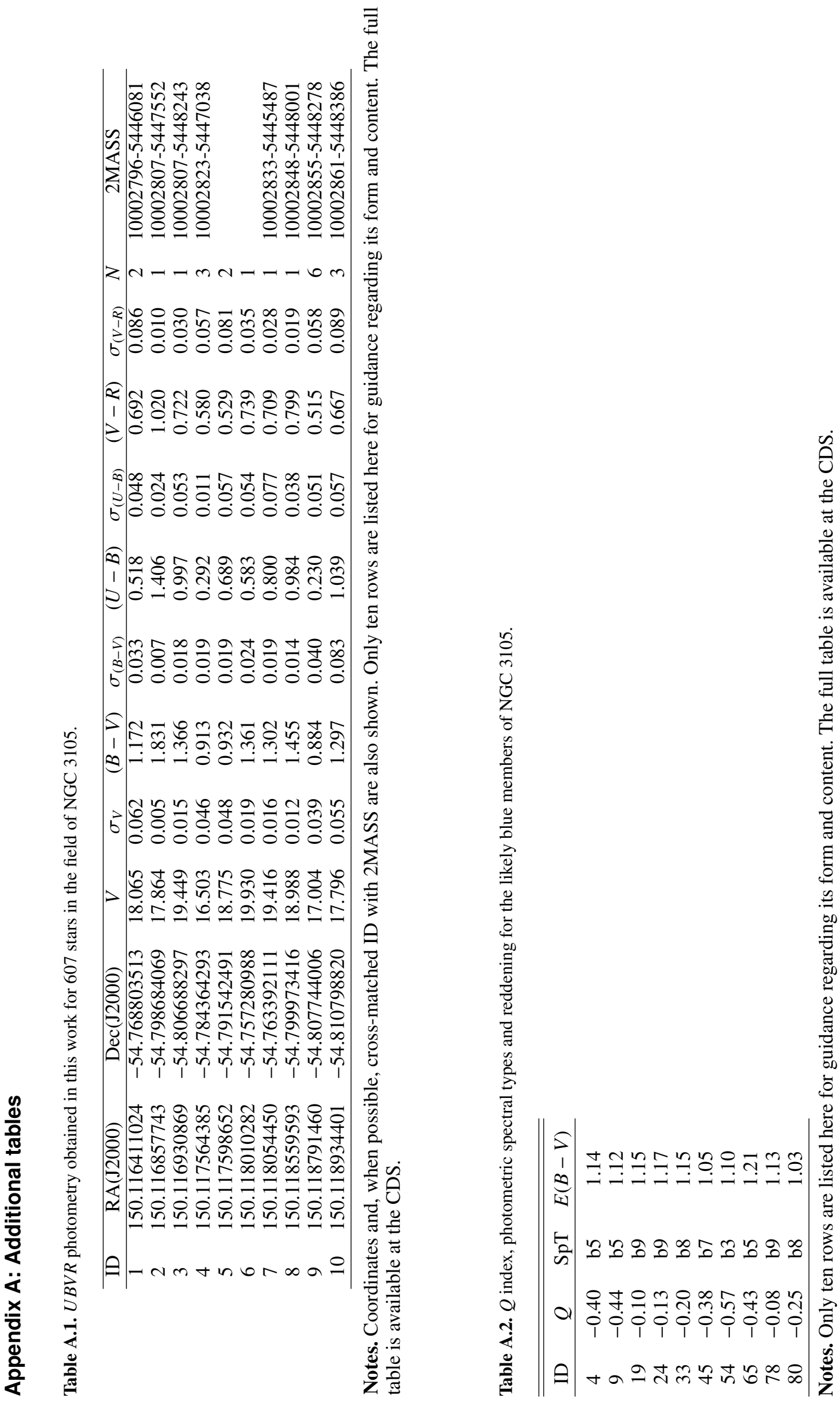

